\title{
PRODUCTS OF COMPLEXES AND FRÉCHET SPACES WHICH ARE MANIFOLDS $\left({ }^{1}\right)$
}

\author{
BY
}

JAMES E. WEST

\begin{abstract}
It is shown that if a locally finite-dimensional simplicial complex is given the "barycentric" metric, then its product with any Fréchet space $X$ of suitably high weight is a manifold modelled on $X$, provided that $X$ is homeomorphic to its countably infinite Cartesian power. It is then shown that if $X$ is Banach, all paracompact $X$-manifolds may be represented (topologically) by such products.
\end{abstract}

In [20] it was established that the product of a separable, infinite-dimensional, Fréchet space and a locally finite simplicial complex is always a paracompact manifold modelled on the Fréchet space. Previously, David Henderson had shown (combining results of [10] and [11]) that each paracompact manifold modelled on a separable, infinite-dimensional, Fréchet space is homeomorphic to the product of that space with a locally finite simplicial complex, so this characterized the products of locally finite, simplicial complexes with separable, infinite-dimensional, Fréchet spaces as precisely the paracompact manifolds modelled on these spaces. In this paper, attention is primarily given to simplicial complexes which are not necessarily locally compact but are given complete metrics and to Fréchet spaces which are not necessarily separable. It is proved (Theorems 3,4 ) that if $K$ is a simplicial complex which is locally finite-dimensional and is given the metric derived from barycentric coordinates (as if the complex were embedded piecewise linearly in a Hilbert space with its vertices all mutually orthogonal and on the unit sphere), then its product with any Fréchet space of suitably large weight which is homeomorphic to its countably infinite Cartesian power is a manifold modelled on that space.

In addition, it is shown, using two other results of Henderson and a suggestion due to him and Israel Berstein, that (Theorem 5) all manifolds which are paracompact and modelled on a Banach space which is homeomorphic to its countably infinite Cartesian power are homeomorphic to products of that space with metric, locally finite-dimensional, simplicial complexes. This leads in turn to a result (Corollary 2) on the splitting of a Banach manifold into the product of a closed

Presented to the Society, January 24, 1970; received by the editors June 11, 1970 and, in revised form, July 8, 1971.

AMS 1969 subject classifications. Primary 5755; Secondary 5525.

Key words and phrases. Fréchet manifold, Banach manifold, metric simplicial complex, homotopy type.

(1) This research was entirely supported by NSF Grant GP-9397. 
submanifold and a Fréchet space when it has the homotopy type of a complex of less weight than the space upon which it is modelled (provided that that space is homeomorphic to its countably infinite Cartesian power). The possibility of obtaining Theorem 5 from something on the order of Theorem 4 was suggested to the author by David Henderson.

Uniform approximations to complete metric spaces. Here the notions of "interior approximation", and "Y-approximation" developed in [20] for the context of compact metric spaces, are extended to complete metric spaces. This is achieved by the expedient of requiring all functions involved to be uniformly continuous. The proofs of Theorems 1 and 2 here are descendants of those given in [20] for Theorems 4.1 and 4.2, respectively.

The term "uniform isomorphism" will here be used to mean "homeomorphism which is uniformly continuous and has a uniformly continuous inverse." "Uniform embedding" will mean "embedding which is a uniform isomorphism onto its image."

If $X$ and $Y$ are complete metric spaces, a uniform interior approximation to $X$ by $Y$ is a sequence $\left\{\zeta_{i}\right\}_{i=1}^{\infty}$ of uniform embeddings of $Y$ in $X$ satisfying

(1) for each positive number $\varepsilon$ there is a positive number $n$ such that $i>n$ implies $\zeta_{i}(Y)$ is $\varepsilon$-dense in $X$,

(2) there is a complete metric space $M$ and a uniform embedding $\alpha$ of $X$ in $M$ such that

(3) for each positive number $\varepsilon$ and for each positive integer $i$, there is a uniform embedding $\beta_{\varepsilon, i}$ of $X$ in $M$ with $d\left(\beta_{\varepsilon, i}(x), \alpha(x)\right) \leqq 2^{-i}$ for all $x$ in $X$ which has the property that

(4) for each positive integer $j$, there is a uniform isomorphism $\gamma_{\varepsilon, i, j}$ of $\alpha \circ \zeta_{i}(Y)$ onto $\beta_{\varepsilon, i} \circ \zeta_{i+j}(Y)$ with $d\left(\gamma_{\varepsilon, i, j} \circ \alpha \circ \zeta_{i}(y), \alpha \circ \zeta_{i}(y)\right) \leqq \varepsilon$ for all $y$ in $Y$.

(Here and later, all metrics will be denoted by " $d$ ".)

If $X$ and $Y$ are compact, $M$ may be taken to be the Hilbert cube. It was shown in [20] that in this case the existence of a uniform interior approximation to $X$ by $Y$ is sufficient to imply that $X$ and $Y$ are homeomorphic. The author does not have an analogue to Lemma 4.1 of [20] in the present case, however, and the iterativity condition defined next serves in its stead.

Let $P$ denote the positive real numbers, $N$ the positive integers, and $S$ the set of all sequences of members of $P \times N$. A uniform interior approximation $\zeta$ to $X$ by $Y$ will be said to be iterative if there is a complete metric space $M$ and a choice, for each $(\sigma, n)$ in $S \times N$, of a subsequence $\zeta_{\sigma, n}=\left\{\zeta_{\sigma, n, i}\right\}_{i \in N}$ of $\zeta$ and embeddings $\alpha_{\sigma, n},\left\{\beta_{\sigma, n, \varepsilon, i}\right\}_{(\varepsilon, i) \in P \times N}$, and $\left\{\gamma_{\sigma, n, \varepsilon, i, j}\right\}_{(\varepsilon, i, j) \in P \times N \times N}$ in $M$ satisfying (1)-(4) for $\zeta_{\sigma, n}$ together with

(i) $\zeta_{\sigma, n+1}$ is a subsequence of $\zeta_{\sigma, n}$,

(ii) $\alpha_{\sigma, n+1}=\beta_{\sigma, n, \sigma(n)}$, and

(iii) if $\sigma(i)=\tau(i)$ for $i=1, \ldots, n$, then the choices are identical for $(\sigma, i)$ and $(\tau, i)$ when $1 \leqq i \leqq n$. 
THEOREM 1. If $X$ and $Y$ are complete metric spaces and there is an iterative uniform interior approximation to $X$ by $Y$, then $X$ and $Y$ are uniformly isomorphic.

Proof. Let $\zeta$ be an iterative uniform interior approximation to $X$ by $Y$, and let $M$ and $\left\{\alpha_{\sigma, n}\right\}_{S \times N},\left\{\beta_{\sigma, n, \varepsilon, i}\right\}_{S \times N \times P \times N}$, and $\left\{\gamma_{\sigma, n, \varepsilon, i, j}\right\}_{S \times N \times P \times N \times N}$ be chosen to satisfy iterativity.

Let $\varepsilon_{1}=\frac{1}{2}, \varepsilon_{2}=\frac{1}{4}$, and $i_{1}=1$. Choose $\sigma_{1}$ in $S$ so that $\sigma_{1}(1)=\left(\frac{1}{2}, 1\right)$. Let $i_{2}>i_{1}$ be a positive integer satisfying the inequality

$$
i_{2}>2-\log _{2}\left(\inf \left\{d\left(\alpha_{\sigma_{1}, 1}(x), \alpha_{\sigma_{1}, 1}\left(x^{\prime}\right)\right) \mid\left(x, x^{\prime}\right) \in X ; d\left(x, x^{\prime}\right) \geqq \frac{1}{4}\right\}\right) .
$$

Now select $\sigma_{2}$ in $S$ with $\sigma_{2}(n)=\left(\varepsilon_{n}, i_{n}\right), n=1,2$. Let $j_{2}$ in $N$ be such that $\zeta_{\sigma_{1}, 1, i_{1}+j_{2}}$ $=\zeta_{\sigma_{2}, 2, i_{2}}$.

Define inductively sequences $\left\{\varepsilon_{n}\right\}_{n=1}^{\infty},\left\{i_{n}\right\}_{n=1}^{\infty},\left\{\sigma_{n}\right\}_{n=1}^{\infty}$, and $\left\{j_{n}\right\}_{n=2}^{\infty}$ of members of $P, N, S$, and $N$, respectively, satisfying

(a) $\varepsilon_{1}, \varepsilon_{2}, i_{1}, i_{2}, \sigma_{1}, \sigma_{2}$, and $j_{2}$ are as selected above,

$$
\begin{aligned}
& i_{n+1}> i_{n}, \\
&> 2-\log _{2}\left(\operatorname { i n f } \left\{d\left(\beta_{\sigma_{n}, n, \sigma_{n}(n)}(x), \beta_{\sigma_{n}, n, \sigma_{n}(n)}\left(x^{\prime}\right)\right) \mid\right.\right. \\
&\left.\left.x, x^{\prime} \in X ; d\left(x, x^{\prime}\right) \geqq 2^{-n-1}\right\}\right), \\
& \varepsilon_{n+1} \leqq \frac{1}{2} \varepsilon_{n}, \\
& \leqq 2^{-3} \inf \left\{d \left(\gamma_{\sigma_{n}, n, \sigma_{n}(n), j_{n}} \circ \cdots \circ \gamma_{\sigma_{n}, 1, \sigma_{n}(1), j_{2}} \circ \alpha_{\sigma_{1}, 1} \circ \zeta_{1}(y),\right.\right. \\
&\left.\gamma_{\sigma_{n}, n, \sigma_{n}(n), j_{n}} \circ \cdots \circ \gamma_{\sigma_{n}, 1, \sigma_{n}(1), j_{2}} \circ \alpha_{\sigma_{1}, 1} \circ \zeta_{1}\left(y^{\prime}\right)\right) \mid \\
&\left.y, y^{\prime} \in Y ; d\left(y, y^{\prime}\right) \geqq 2^{-n-1}\right\},
\end{aligned}
$$

(d) $\sigma_{n+1}(m)=\left(\varepsilon_{m}, i_{m}\right), m=1, \ldots, n+1$, and

(e) $\zeta_{\sigma_{n}, n, i_{n}+j_{n+1}}=\zeta_{\sigma_{n+1}, n+1, i_{n+1}}$.

Now the sequences $\sigma=\left\{\left(\varepsilon_{n}, i_{n}\right)\right\}_{n \in N}$ and $\left\{j_{n}\right\}_{n \in N}$ define sequences $\left\{f_{n}=\beta_{\sigma, n, \sigma(n)}\right\}_{n \in N}$ and $\left\{g_{n}=\gamma_{\sigma, n, \sigma(n), j_{n+1}} \circ \cdots \circ \gamma_{\sigma, 1, \sigma(1), j_{2}} \circ \alpha_{\sigma, 1} \circ \zeta_{1}\right\}_{n \in N}$ of embeddings of $X$ and $Y$, respectively, in $M$. These, in fact, converge uniformly to uniform isomorphisms onto the same subset of $M$.

First, $\left\{f_{n}\right\}_{n \in N}$ and $\left\{g_{n}\right\}_{n \in N}$ are uniformly Cauchy because of the initial restrictions of (b) and (c), respectively, together with (3) and (4), respectively. Hence, they converge to uniformly continuous functions $f$ and $g$ from $X$ and $Y$ into $M$, respectively. However, the second inequalities of (b) and (c) guarantee that $f$ and $g$ are uniform embeddings.

Second, $f(X)$ and $g(Y)$ are dense in each other. This may be seen as follows: For each $\varepsilon$ in $P$, there is an $n_{1}$ in $N$ such that $n \geqq n_{1}$ implies $d\left(f(x), f_{n}(x)\right)<\varepsilon / 5$ and $d\left(g(y), g_{n}(y)\right)<\varepsilon / 5$ for each $x$ in $X$ and $y$ in $Y$. Because $f_{n_{1}}$ is a uniform embedding, (1) gives an $n_{2}$ in $N$ with $n_{2} \geqq n_{1}$ such that $n \geqq n_{2}$ implies $f_{n_{1}} \circ \zeta_{n}(Y)$ is $\varepsilon / 5$-dense in $f_{n_{1}}(X)$. Also, there is an $n_{3}$ in $N, n_{3} \geqq n_{2}$, such that $\zeta_{n_{3}}=\zeta_{\sigma, n_{2}, i_{n_{2}}}$. Now, if $x \in X$, then 
there is a $y \in Y$ such that $d\left(f_{n_{1}}(x), f_{n_{1}} \circ \zeta_{n_{3}}(y)\right) \leqq \varepsilon / 5$, and since $g_{n_{2}}(Y)$ $=f_{n_{2}} \circ \zeta_{\sigma, n_{2}, i_{n_{2}}}(Y)$, there is a $y^{\prime}$ in $Y$ with $g_{n_{2}}\left(y^{\prime}\right)=f_{n_{2}} \circ \zeta_{\sigma, n_{2}, i_{n_{2}}}(y)$. Then

$$
\begin{aligned}
d\left(f(x), g\left(y^{\prime}\right)\right) \leqq & d\left(f(x), f_{n_{1}}(x)\right)+d\left(f_{n_{1}}(x), f_{n_{1}} \circ \zeta_{n_{3}}(y)\right) \\
& +d\left(f_{n_{2}} \circ \zeta_{n_{3}}(y), f_{n_{1}} \circ \zeta_{n_{3}}(y)\right)+d\left(g_{n_{2}}\left(y^{\prime}\right), f_{n_{2}} \circ \zeta_{n_{3}}(y)\right) \\
& +d\left(g_{n_{2}}\left(y^{\prime}\right), g\left(y^{\prime}\right)\right) \\
< & \varepsilon / 5+\varepsilon / 5+2 \varepsilon / 5+0+\varepsilon / 5=\varepsilon,
\end{aligned}
$$

so $g(Y)$ is dense in $f(X)$. On the other hand, as $g_{n}(Y) \subset f_{n}(X)$ for each $n$ and $\left\{g_{n}\right\}_{n \in N}$ converges uniformly to $g$ while $\left\{f_{n}\right\}_{n \in N}$ converges uniformly to $f, f(X)$ is dense in $g(Y)$.

Finally, since $f$ and $g$ are uniform embeddings of complete metric spaces, their images are closed, hence equal, so $f^{-1} \circ g$ is a uniform isomorphism of $Y$ onto $X$.

If $X$ and $Y$ are complete metric spaces, a closed subset $Z$ of $X \times Y$ will be called a uniform $Y$-approximation to $X$ provided that there exists a complete metric space $M$ and, for every $\varepsilon$ in $P$, a uniform embedding $g_{\varepsilon}$ of $X \times Y$ in $Z \times M \subset X \times Y \times M$ with, for each $x$ in $X \times Y, d\left(p_{1} \circ g_{\varepsilon}(x), p_{1}(x)\right) \leqq \varepsilon$ such that if $\bar{g}_{\varepsilon}=\left(p_{1} \circ g_{\varepsilon} \circ\left(p_{1}, p_{2}\right)\right.$, $\left.p_{2} \circ g_{\varepsilon} \circ\left(p_{1}, p_{2}\right), p_{3}, p_{3} \circ g_{\varepsilon} \circ\left(p_{1}, p_{2}\right)\right): X \times Y \times Y \rightarrow Z \times Y \times M \subset X \times Y \times Y \times M$ and if $Z_{i}=\left(p_{1}, p_{i+1}\right)^{-1}(Z), i=1,2$, then there is a uniform isomorphism $h_{\varepsilon}: \bar{g}_{\varepsilon}\left(Z_{2}\right) \rightarrow Z_{1}$ with $d\left(\left(p_{1}, p_{2}\right) \circ h_{\varepsilon}(x),\left(p_{1}, p_{2}\right)(x)\right) \leqq \varepsilon$ for all $x$ in $\bar{g}_{\varepsilon}\left(Z_{2}\right)$. (Here, $p_{i}$ is the projection onto the $i$ th factor, and $Z$ is always regarded as a subset of $X \times Y$. The metric on finite products will always be the sum of the coordinate metrics. In the following, infinite products of complete metrics appear, and it will be convenient to make the convention that given a collection $\left\{A_{n}\right\}_{n \in N}$ of metric spaces with uniformly bounded diameters, the metric for $\prod_{n \in N} A_{n}$ will be given by $d(x, y)=\sum_{n \in N} 2^{-i-1} d\left(p_{n}(x), p_{n}(y)\right)$. Also, given a space $Y$, the symbol $Y^{\infty}$ will denote the product of a countably infinite set of copies of $Y$ indexed by $N$.)

THEOREM 2. If $X$ and $Y$ are complete metric spaces and there is a uniform $Y$ approximation $Z$ to $X$, then $X \times Y^{\infty}$ and $Z \times Y^{\infty}$ are uniformly isomorphic.

Proof. Let $M$ be a complete metric space as in the definition of uniform $Y$ approximation. For convenience, it will be assumed that the diameters of $X, Y$, and $M$ are no greater than one. (This may be done because if $A$ is any metric space, the new metric $\rho(x, y)=\min \{d(x, y), 1\}$ gives diameter less than or equal to one, and the identity function is a uniform isomorphism.)

Let $\zeta=\left\{\zeta_{i}\right\}_{i \in N}$, where $\zeta_{i}: Z \times Y^{\infty} \rightarrow X \times Y^{\infty}$ is given by $\zeta_{i}=\left(p_{1},\left(p_{1} \circ p_{3}, p_{2} \circ p_{3}, \ldots\right.\right.$, $\left.p_{2 i-1} \circ p_{3}, p_{2}, p_{2 i} \circ p_{3}, p_{2 i+1} \circ p_{3}, \ldots\right)$ ). (The notation supposes $Z \times Y^{\infty} \subset X \times Y$ $\times Y^{\infty}$.) It will be shown to be an iterative uniform interior approximation.

Fix a point $m$ in $M$ and let $\alpha$ embed $X \times Y^{\infty}$ in $X \times Y^{\infty} \times M^{\infty}$ by the formula

$$
(x, y) \rightarrow(x, y,(m, m, m, \ldots))
$$


In order to define $\left\{\beta_{\varepsilon, i}\right\}_{P \times N}$ and $\left\{\gamma_{\varepsilon, i, j}\right\}_{P \times N \times N}$, a "rotation" of $X \times Y^{\infty} \times M^{\infty}$ is necessary. Let $k: P \times N \rightarrow N$ be a function such that $k(\varepsilon, i) \geqq i+2$ and $2-\log _{2}(\varepsilon)$, and for each $\varepsilon$ in $P$ and $i$ in $N$, define $\varphi_{\varepsilon, i}: X \times Y^{\infty} \times M^{\infty} \rightarrow X \times Y^{\infty} \times M^{\infty}$ by

$(\varphi, 1) \quad p_{j} \circ \varphi_{\varepsilon, i}=p_{j}$, if $j=1,3$,

$(\varphi, 2) \quad p_{j} \circ p_{2} \circ \varphi_{\varepsilon, i}=p_{j} \circ p_{2}$, if $j=1, \ldots, 2 i$,

$(\varphi, 3) \quad p_{2 k(\varepsilon, i)+2 j} \circ p_{2} \circ \varphi_{\varepsilon, i}=p_{2 i+2 j} \circ p_{2}$

$(\varphi, 4) \quad p_{2 i+2 j} \circ p_{2} \circ \varphi_{\varepsilon, i}=p_{2 i+2 j-1} \circ p_{2}$, if $j=1, \ldots, k(\varepsilon, i)-i$, and

$(\varphi, 5) \quad p_{2 i+2 j-1} \circ p_{2} \circ \varphi_{\varepsilon, i}=p_{2 k(\varepsilon, i)+2 j-1} \circ p_{2}$.

For each $(\varepsilon, i)$ in $P \times N$, define $\beta_{\varepsilon, i}$ by

$(\beta, 1) \quad p_{1} \circ \beta_{\varepsilon, i}=p_{1} \circ g_{2}-k(\varepsilon, i) \circ\left(p_{1}, p_{2 i} \circ p_{2}\right)$,

$(\beta, 2) \quad p_{j} \circ p_{2} \circ \beta_{\varepsilon, i}=p_{j} \circ p_{2} \circ \varphi_{\varepsilon, i}$, for $j \neq 2 i$,

$(\beta, 3) \quad p_{2 i} \circ p_{2} \circ \beta_{\varepsilon, i}=p_{2} \circ g_{2}^{-k(\varepsilon, i)} \circ\left(p_{1}, p_{2 i} \circ p_{2}\right)$,

$(\beta, 4) \quad p_{j} \circ p_{3} \circ \beta_{\varepsilon, i}=m$, for $j \neq k(\varepsilon, i)$, and

$(\beta, 5) \quad p_{k(\varepsilon, i)} \circ p_{3} \circ \beta_{\varepsilon, i}=p_{3} \circ g_{2}-k(\varepsilon, i) \circ\left(p_{1}, p_{2 i} \circ p_{2}\right)$.

(That is, $\beta_{\varepsilon, i}$ is $\varphi_{\varepsilon, i} \circ \alpha$ followed by the action of $g_{2^{-k(\varepsilon, i)}}$ in the coordinates $X, Y_{2 i}$, and $M_{k(\varepsilon, i)}$.)

For each $(\varepsilon, i, j)$ in $P \times N \times N$, define

$$
\gamma_{\varepsilon, i, j}: \alpha \circ \zeta_{i}\left(Z \times Y^{\infty}\right)=\left(p_{1}, p_{2 i} \circ p_{2}, p_{3}\right)^{-1}(Z \times\{(m, m, \ldots)\}) \rightarrow X \times Y^{\infty} \times M^{\infty}
$$

by

$\left.(\gamma, 1) \quad p_{1} \circ \gamma_{\varepsilon, i, j}=p_{1} \circ h_{2}^{-k(\varepsilon, i)}\right)^{-1} \circ\left(p_{1}, p_{2 i} \circ p_{2}, p_{2 k(\varepsilon, i)+2 j} \circ p_{2}\right)$,

$(\gamma, 2) \quad p_{n} \circ p_{2} \circ \gamma_{\varepsilon, i, j}=p_{n} \circ p_{2}$, for $n \neq 2 i, 2 k(\varepsilon, i)+2 j$,

$(\gamma, 3) \quad p_{2 i} \circ p_{2} \circ \gamma_{\varepsilon, i, j}=p_{2} \circ h_{2}-k(\varepsilon, i)-1 \circ\left(p_{1}, p_{2 i} \circ p_{2}, p_{2 k(\varepsilon, i)+2 j} \circ p_{2}\right)$,

$\left.(\gamma, 4) \quad p_{2 k(\varepsilon, i)+2 j} \circ p_{2} \circ \gamma_{\varepsilon, i, j}=p_{3} \circ h_{2}-k(\varepsilon, i)\right)^{-1} \circ\left(p_{1}, p_{2 i} \circ p_{2}, p_{2 k(\varepsilon, i)+2 j} \circ p_{2}\right)$,

$(\gamma, 5) \quad p_{n} \circ p_{3} \circ \gamma_{\varepsilon, i, j}=m, n \neq k(\varepsilon, i)$, and

$(\gamma, 6) \quad p_{k(\varepsilon, i)} \circ p_{3} \circ \gamma_{\varepsilon, i, j}=p_{4} \circ h_{2}^{-k(\varepsilon, i)}-1 \circ\left(p_{1}, p_{2 i} \circ p_{2}, p_{2 k(\varepsilon, i)+2 j} \circ p_{2}\right)$.

(That is, $\gamma_{\varepsilon, i, j}$ is the action of $h_{2}^{-k(\varepsilon, i)}{ }^{-1}$ on the $X, Y_{2 i}, Y_{2 k(\varepsilon, i)+2 j}$, and $M_{k(\varepsilon, i)}$ coordinates.)

These functions establish that $\zeta$ is a uniform interior approximation to $X \times Y^{\infty}$ by $Z \times Y^{\infty}$. For each $(\varepsilon, i)$ in $P \times N$ and $x$ in $X \times Y^{\infty}$,

$$
\begin{aligned}
& d\left(\beta_{\varepsilon, i}(x), \alpha(x)\right) \leqq d\left(p_{1} \circ g_{2^{-k(\varepsilon, i)}}\left(p_{1}, p_{2 i} \circ p_{2}\right)(x), p_{1}(x)\right)+\operatorname{dia}\left(\prod_{j \geq 2 i} Y_{j}\right)+\operatorname{dia}\left(M_{k(\varepsilon, i)}\right) \\
& \leqq 2^{-k(\varepsilon, i)}+2^{-2 i}+2^{-k(\varepsilon, i)-1} \leqq 2^{-i-1}+2^{-2 i} \leqq 2^{-i} \text {. }
\end{aligned}
$$

If $(\varepsilon, i, j)$ is in $P \times N \times N$, then for $y$ in $\alpha \circ \zeta_{i}\left(Z \times Y^{\infty}\right)$,

$$
\begin{aligned}
& d\left(\gamma_{\varepsilon, i, j}(y), y\right) \leqq d\left(p_{1} \circ h_{2}^{-k(\varepsilon, i)}-1 \circ\left(p_{1}, p_{2 i} \circ p_{2}, p_{2 k(\varepsilon, i)+2 j} \circ p_{2}\right)(y), p_{1}(y)\right) \\
& +d\left(p_{2 i} \circ p_{2} \circ h_{2}-k(\varepsilon, i)-1 \circ\left(p_{1}, p_{2 i} \circ p_{2}, p_{2 k(\varepsilon, i)+2 j} \circ p_{2}\right)(x), p_{2 i} \circ p_{2}(x)\right) \\
& +\operatorname{dia}\left(Y_{2 k(\varepsilon, i)+2 j}\right)+\operatorname{dia}\left(M_{k(\varepsilon, i)}\right) \\
& <2^{-k(\varepsilon, i)}+2^{-k(\varepsilon, i)}+2^{-k(\varepsilon, i)}=3 \cdot 2^{-k(\varepsilon, i)}<\varepsilon .
\end{aligned}
$$

(The first $2^{-k(\varepsilon, i)}$ term is contributed by the first two terms of the preceding part of the inequality because of the restriction placed on $h_{2}-k(\varepsilon, i)$ in the definition of 
uniform $Y$-approximation.) It is clear that all functions involved are uniform isomorphisms, so there only remains to verify that $\gamma_{\varepsilon, i, j}\left(\alpha \circ \zeta_{i}\left(Z \times Y^{\infty}\right)\right)$ $=\beta_{\varepsilon, i} \circ \zeta_{i+j}\left(Z \times Y^{\infty}\right)$. The coordinates defining $\zeta_{i+j}\left(Z \times Y^{\infty}\right)$ are $X$ and $Y_{2(i+j)}$, however, and $(\varphi, 3)$ shows that $\varphi_{\varepsilon, i} \circ \alpha \circ \zeta_{i+j}\left(Z \times Y^{\infty}\right)=\alpha \circ \zeta_{2 k(\varepsilon, i)+2,}\left(Z \times Y^{\infty}\right)$. Therefore, following the parenthetical remark after the definition of $\beta_{\varepsilon, i}, \beta_{\varepsilon, i}$ $\circ \zeta_{i+j}\left(Z \times Y^{\infty}\right)$ is the image under the application of $g_{2^{-k(\varepsilon, i)}}$ in the $X$ and $Y_{2 i}$ coordinates, moving into the $M_{k(\varepsilon, i)}$ coordinate, of $\alpha \circ \zeta_{2 k(\varepsilon, i)+2 j}\left(Z \times Y^{\infty}\right)$, which is also the image under the application of $h_{2}^{-k(\varepsilon, i)}{ }^{-1}$ to the $X, Y_{2 i}$, and $Y_{2 k(\varepsilon, i)+2 j}$ coordinates, moving into the $M_{k(\varepsilon, i)}$ coordinate, which function is $\gamma_{\varepsilon, i, j}$.

In order to demonstrate the iterativity of $\zeta$, let $r: X \times Y^{\infty} \times M^{\infty} \rightarrow X \times Y^{\infty}$ $\times\{(m, m, \ldots)\}$ be the obvious retraction, and for $i$ in $N$, let $\pi_{i}: X \times Y^{\infty} \times M^{\infty}$ $\rightarrow X \times Y^{\infty} \times \prod_{j \neq i} M_{j}$ be the projection off the $M_{i}$ coordinate. Now let $\nu: P \times N \times N \rightarrow N$ and $\eta: P \times N \times P \rightarrow P$ be functions such that (1) for $x$ and $y$ in $\alpha\left(X \times Y^{\infty}\right)$ with $d(x, y) \leqq 2^{1-v(\varepsilon, m, i)}, d\left(\beta_{\varepsilon, m} \circ \alpha^{-1}(x), \beta_{\varepsilon, m} \circ \alpha^{-1}(y)\right) \leqq 2^{-i-1}$, (2) $\eta(\varepsilon, m, \delta)<\delta / 2$, (3) for $x$ and $y$ in $\alpha\left(X \times Y^{\infty}\right)$ with $d(x, y) \leqq 2 \eta(\varepsilon, m, \delta)$, $d\left(\beta_{\varepsilon, m} \circ \alpha^{-1}(x), \beta_{\varepsilon, m} \circ \alpha^{-1}(y)\right) \leqq \delta / 2$, and (4) $k(\eta(\varepsilon, m, \delta), \nu(\varepsilon, m, i))>k(\varepsilon, m)$. Now for each $\sigma$ in $S$ let $\alpha_{\sigma, 1}=\alpha, \beta_{\sigma, 1, \varepsilon, i}=\beta_{\varepsilon, i}$, and $\gamma_{\sigma, 1, \varepsilon, i, j}=\gamma_{\varepsilon, i, j}$, and let

$$
\begin{aligned}
\beta_{\sigma, 2, \delta, i}= & \left(\pi_{k(\eta(\sigma(1), \delta), v(\sigma(1), i))} \circ \beta_{\sigma(1)} \circ \alpha^{-1} \circ r, p_{k(\eta(\sigma(1), \delta), v(\sigma(1), i))} \circ p_{3}\right) \\
& \circ \beta_{\eta(\sigma(1), \delta), v(\sigma(1), i)}: X \times Y^{\infty} \rightarrow\left(X \times Y^{\infty} \times \prod_{j \neq k(\eta(\sigma(1), \delta), v(\sigma(1), i))} M_{j}\right) \\
& \times M_{k(\eta(\sigma(1), \delta), v(\sigma(1), i))}=X \times Y^{\infty} \times M^{\infty} .
\end{aligned}
$$

Also, let

$$
\begin{aligned}
\gamma_{\sigma, 2, \delta, i, j}= & \left(\pi_{k(\eta(\sigma(1), \delta), v(\sigma(1), i))} \circ \beta_{\sigma(1)} \circ \alpha^{-1} \circ r, p_{k(\eta(\sigma(1), \delta), v(\sigma(1), i))} \circ p_{3}\right) \\
& \circ \gamma_{\eta(\sigma(1), \delta), v(\sigma(1), i), v(\sigma(1), i+j)-v(\sigma(1), i)} \circ \alpha \circ \beta_{\sigma(1)}^{-1} .
\end{aligned}
$$

Then with $\alpha_{\sigma, 2}=\beta_{\sigma(1)}$ and $\zeta_{\sigma, 2}=\left\{\zeta_{v(\sigma(1), i)}\right\}_{i \in N}$, this choice satisfies (1)-(4) and(i)-(iii) (with $n=1,2$ ). An induction completes the construction.

Since $\zeta$ is an iterative uniform interior approximation to $X \times Y^{\infty}$ by $Z \times Y^{\infty}$, Theorem 1 applies to finish the proof.

Some technical lemmas. In the following, proofs are abbreviated to constructions, since the verifications that the constructions do work are straightforward.

Let $I^{\infty}=\prod_{i \in N}[0,1]_{i}$ and denote by $\mathscr{H}_{0}\left(I^{\infty}\right)$ those homeomorphisms of $I^{\infty}$ onto itself which preserve $p_{i}^{-1}(0)$ for each $i \in N$. For any space $X$, let $\mathscr{F}(X)$ be the functions from $X$ into itself; if $X$ is a uniform space, let $\mathscr{U}(X)$ denote the uniform isomorphisms of $X$ onto itself. If $\left\{E_{i}\right\}_{i \in N}$ is any countably infinite collection of Banach spaces, let $\nu: \prod_{i \in N} E_{i} \rightarrow \prod_{i \in N} R_{i}$, where $R_{i}$ is the real numbers, be defined by $p_{i} \circ \nu(x)$ $=\left\|p_{i}(x)\right\|$ for all $x$ in $\prod_{i \in N} E_{i}$ and $i$ in $N$. Also, let $\xi: \prod_{i \in N} E_{i} \rightarrow \prod_{i \in N} E_{\imath}$ be defined by

$$
\begin{aligned}
p_{i} \circ \xi(x) & =p_{i}(x) /\left\|p_{i}(x)\right\| & & \text { if } p_{i}(x) \neq 0 \\
& =0 & & \text { if } p_{i}(x)=0
\end{aligned}
$$


for all $x$ in $\prod_{i \in N} E_{i}$ and $i$ in $N$, and let $\mu: \prod_{i \in N} R_{i} \times \prod_{i \in N} E_{i} \rightarrow \prod_{i \in N} E_{i}$ be coordinatewise multiplication. Finally, denote the closed unit ball of $E_{i}$ centered at the origin by $B_{i}$, and define $\Phi: \mathscr{F}\left(I^{\infty}\right) \rightarrow \mathscr{F}\left(\prod_{i \in N} E_{i}\right)$ by $\Phi(f)=\mu \circ(f \times \mathrm{id}) \circ(\nu, \xi)$.

Lemma 1. $\Phi\left(\mathscr{H}_{0}\left(I^{\infty}\right)\right) \subset \mathscr{U}\left(\prod_{i \in N} B_{i}\right)$; furthermore, the constants of uniform continuity of $\Phi(f)$ and $\Phi(f)^{-1}$ depend only on $f$, not $\left\{E_{i}\right\}_{i \in N}$.

LEMMA 2. There is a member $\lambda$ of $\mathscr{U}\left(\prod_{i \in N} B_{i}\right)$ and a collection $\left\{\delta_{i}\right\}_{i>1} \subset(0,1)$ such that $\lambda\left(p_{1}^{-1}\left(S_{1}\right)\right) \subset S_{1} \times \prod_{i>1} \delta_{i} B_{i}$, where $S_{1}$ is the unit sphere of $B_{1}$. The collection $\left\{\delta_{i}\right\}_{i>1}$ is independent of $\left\{E_{i}\right\}_{i \in N}$, as are the constants of uniform continuity for $\lambda$ and $\lambda^{-1}$.

Proof. Theorem 6.1 of [1] asserts the existence of a homeomorphism $h$ of $I^{\infty}$ onto itself with the property that $h\left(p_{1}^{-1}(0)\right) \subset\{0\} \times \prod_{i>1}(0,1)_{i}$. An examination of the proof gives quickly that it may be modified to yield the following: "There is a member $\theta$ of $\mathscr{H}_{0}\left(I^{\infty}\right)$ such that $\theta\left(p_{1}^{-1}(1)\right) \subset\{1\} \times \prod_{i>1}[0,1)_{i}$." Let $\lambda=\Phi(\theta)$.

LemMA 3. Let $J$ be any infinite subset of $N$, and $M$, any infinite subset of $J$ for which $J \backslash M$ is also infinite. If $\left\{\delta_{j}\right\}_{j \in J} \subset(0,1)$, there is an element $\varphi$ of $\mathscr{U}\left(\prod_{i \in N} B_{i}\right)$ carrying $\bigcap_{j \in J} p_{j}^{-1}\left(\delta_{j} B_{j}\right)$ into $\left[\bigcap_{j \in J} p_{j}^{-1}\left(\varepsilon_{j} B_{j}\right)\right] \cap\left[\bigcap_{m \in M} p_{m}^{-1}(0)\right]$, for some collection $\left\{\varepsilon_{j}\right\}_{j \in J} \subset(0,1)$. Furthermore, $\varphi$ may be required to have the property that $p_{i} \circ \varphi=p_{i}$ if $i \notin J$.

Proof. This, as Lemma 2, is a generalization of a result in [1]. The proof is analogous to that in [1] except for one or two points.

Let $\left\{J_{m}\right\}_{m \in M}$ be a collection of pairwise disjoint infinite subsets of $J \backslash M$, and denote the members of $J_{m}$ by $j(m, i)$, where $j(m, i) \geqq i$ for all $m$. For each $m$ and $i$ in $N$, let $\psi_{m, i}:\left[0, \delta_{j(m, i)}, 1\right] \rightarrow\left[0,2^{-i-4}\left(1-\delta_{m}\right), 1\right]$ be the indicated orientationpreserving piecewise-linear homeomorphism, and let $\chi_{m, i} \in \mathscr{U}\left(B_{m} \times B_{j(m, i)}\right)$ be $\left(p_{1},\left(\chi_{m, i} \circ\|\cdot\| \circ p_{2}\right) \cdot p_{2}\right)$. Let $\varphi_{m, i} \in \mathscr{U}\left(B_{m} \times B_{j(m, i)}\right)$ be defined by

$$
\begin{aligned}
\varphi_{m, i} & =\left(p_{1}, p_{2}+\left[(1-\|\cdot\|) \circ p_{2}\right] \cdot p_{1},\right. \\
& =\left(p_{1}, p_{2}+\left(\left[(1-\|\cdot\|) \circ p_{2}\right]\left[(1-\|\cdot\|) \circ p_{1}\right] /\left(1-\delta_{m}\right)\right) \cdot p_{1},\right. \\
& \text { on }\left(B_{m} \mid \delta_{m} B_{m}\right) \times B_{j(m, i)} .
\end{aligned}
$$

Now let $\psi_{m, i}=\varphi_{m, i} \circ \chi_{m, i}$. It has the properties that it changes the $B_{m}$-coordinate of no point, that it carries $\delta_{m} \cdot B_{m} \times \delta_{j(m, i)} \cdot B_{j(m, i)}$ into $\delta_{m} \cdot B_{m} \times \varepsilon_{j(m, i)} \cdot B_{j(m, i)}$ (where $\left.\varepsilon_{j(m, i)}=2^{-i-4}\left(1-\delta_{m}\right)^{2}+\delta_{m}\right)$, so that the projection into $B_{j(m, i)}$ carries the image onto $\varepsilon_{j(m, i)} \cdot B_{j(m, i)}$, and that for $x$ and $y$ in $\delta_{m} \cdot B_{m} \times \delta_{j(m, i)} \cdot B_{j(m, i)}$ with $\left\|p_{1}(x)-p_{1}(y)\right\|$ $\geqq 2^{-i},\left\|p_{2} \circ \psi_{m, i}(x)-p_{2} \circ \psi_{m, i}(y)\right\|>2^{-j-1}$. Define $\psi \in \mathscr{U}\left(\prod_{i \in N} B_{i}\right)$ to be that element defined by $\left\{\psi_{m, i}\right\}_{(m, i) \in M \times N}$. (More precisely, let each $\psi_{m, i}$ be extended to a uniform isomorphism of $\prod_{i \in N} B_{i}$ in the natural way, and let $\psi_{m}=\operatorname{limit}_{i \rightarrow \infty} \psi_{m, i} \circ \ldots \circ \psi_{m, 1}$ for each $m$ in $M$. Then $\psi$ may be defined to be $\operatorname{limit}_{n \rightarrow \infty} \psi_{m(n)} \circ \ldots \circ \psi_{m(1)}$, where $M=\{m(n)\}_{n \in N}$ is an enumeration of $M$.) 
Here, let $\pi_{m}: \delta_{m} \cdot B_{m} \times \prod_{i \in N} B_{j(m, i)} \rightarrow \prod_{i \in N} B_{j(m, i)}$ be the projection. As noted above, $\pi_{m} \circ \varphi_{m}\left(\delta_{m} \cdot B_{m} \times \prod_{i \in N} \delta_{j(m, i)} \cdot B_{j(m, i)}\right)=\prod_{i \in N} \varepsilon_{j(m, i)} \cdot B_{j(m, i)}$; further,

$$
\pi_{m} \circ \varphi_{m} \mid \delta_{m} \cdot B_{m} \times \prod_{i \in N} \delta_{j(m, i)} \cdot B_{j(m, i)}
$$

is a uniform isomorphism. Thus,

$$
\bar{\omega}_{m}=p_{1} \circ\left(\pi_{m} \mid \varphi_{m}\left(\delta_{m} \cdot B_{m} \times \prod_{i \in \bar{N}} \delta_{j(m, i)} \cdot B_{j(m, i)}\right)\right)^{-1}
$$

is a uniformly continuous map of $\prod_{i \in N} \varepsilon_{j(m, i)} \cdot B_{j(m, i)}$ onto $\delta_{m} \cdot B_{m}$. Let $\rho_{m, i}$ be the radial retraction of $B_{j(m, i)}$ onto $\varepsilon_{j(m, i)} \cdot B_{j(m, i)}$, and let $\rho_{m}=\prod_{i \in N} \rho_{j(m, i)}$. The function $\bar{\omega}_{m} \circ \rho_{m}$ is a uniformly continuous map of $\prod_{i \in N} B_{j(m, i)}$ into $\delta_{m} \cdot B_{m}$. Define $\omega_{m}$ in $\mathscr{U}\left(B_{m} \times \prod_{i \in N} B_{j(m, i)}\right)$ to be $\left(p_{1}+\left[(1-\|\cdot\|) \circ p_{1}\right] \cdot \bar{\omega}_{m} \circ \rho_{m} \circ \pi_{m}, \pi_{m}\right)^{-1}$, and extend it naturally to a member of $\mathscr{U}\left(\prod_{i \in N} B_{i}\right)$. Let $\omega=\operatorname{limit}_{n \rightarrow \infty} \omega_{m(n)} \circ \cdots \circ \omega_{m(1)}$. The composition $\omega \circ \varphi$ is the desired function, and for $j \in J \backslash\left[M \cup\left(\bigcup_{m \in M} J_{m}\right)\right], \varepsilon_{j}$ may be had to be $\delta_{j}$.

LEMMA 4. If $E$ is a Banach space with (closed) unit ball $B$ and unit sphere $S$, then for each $\varepsilon$ in $(0,1)$ there exists a collection $\left\{H_{s}\right\}_{s \in S}$, of closed, codimension one hyperplanes of $E$ with $s \notin H_{s}$, a collection $\left\{W_{s}\right\}_{s \in S}$, of closed, symmetric, bounded, convex neighborhoods of the origin in the hyperplanes, $W_{s} \subset H_{s}$, and a collection $\left\{\alpha_{s}\right\}_{s \in S}$ of homeomorphisms of $B, \alpha_{s}: B \rightarrow W_{s}+[-1,1] \cdot s$, such that

(1) $\alpha_{s}(S)=\left[W_{s}+\{s,-s\}\right] \cup\left[W_{s} \mid[0,1) \cdot W_{s}+[-1,1] \cdot s\right]$,

(2) $\alpha_{s}( \pm s)= \pm s$

(3) there exists $a \delta$ in $(0,1)$ for which $\alpha_{s}^{-1}\left(W_{s} \pm[1-\delta, 1] \cdot s\right) \subset \varepsilon \cdot B \pm s$, and

(4) $\left\{\alpha_{s}\right\}_{s \in S} \cup\left\{\alpha_{s}^{-1}\right\}_{s \in S}$ is uniformly equicontinuous.

Proof. (Here, $A+B$ denotes $\{a+b \mid a \in A, b \in B\}$, and $C \cdot A$ denotes $\{c \cdot a \mid c \in C$, $a \in A\}$.)

For each $s$ in $S$ select, by the Hahn-Banach Theorem, a continuous, real-valued, linear functional $f_{s}: E \rightarrow R$ of norm one sending $s$ to one. Let $H_{s}=f_{s}^{-1}(0)$ and let $W_{s}=(\varepsilon / 8) \cdot\left[\left(I-f_{s}(\cdot) \cdot s\right)(B)\right]$. Since $W_{s}+[-1,1] \cdot s$ is a closed, convex, bounded, symmetric neighborhood of the origin in $E$, the Minkowski functional $x \rightarrow p_{s}(x)$ $=\inf \left\{t>0 \mid x \in t\left(W_{s}+[-1,1] \cdot s\right)\right\}$ of $W_{s}+[-1,1] \cdot s$ is a norm for $E$ which is equivalent to the original one, $\|\cdot\|$. In fact, $(4 /(4+\varepsilon))\|x\| \leqq p_{s}(x) \leqq(8 / \varepsilon)\|x\|$ for all $x$ in $E, s$ in $S$. The function $\alpha_{s}: B \rightarrow W_{s}+[-1,1] \cdot s$ may be defined by

$$
\begin{aligned}
\alpha_{s}(x) & =\left(\|x\| / p_{s}(x)\right) x & & \text { if } x \neq 0, \\
& =0 & & \text { if } x=0,
\end{aligned}
$$

and for this choice, $\varepsilon / 7$ will do for $\delta$.

Throughout, the phrase "metric simplicial complex" will denote a simplicial complex with the topology generated by the Euclidean metric on the barycentric representation of its cells. (That is, if the complex $K$ is regarded as a piecewise linear subset of some Hilbert space with the property that all its vertices are mem- 
bers of a collection of mutually orthogonal unit vectors, then the metric is just that induced by the norm of the Hilbert space.) The symbol st $(v, K)$ denotes the subcomplex of $K$ composed of those simplices of $K$ containing $v$.

Assuming the terminology of Lemma 4, suppose that $K$ is a finite-dimensional, metric, simplicial complex, that $A \subset S$, that $\left\{L_{s}\right\}_{s \in A}$ is a collection of subcomplexes of $K$ indexed by $A$, that there is a vertex $v$ of $K$ such that $K=\operatorname{st}(v, K)$ and $L_{s}=$ st $\left(v, L_{s}\right)$, for all $s$ in $A$, and that $\left\{\mu_{s}\right\}_{s \in A}$ is a collection, uniformly equicontinuous when taken together with $\left\{\mu_{s}^{-1}\right\}_{s \in A}$, of homeomorphisms $\mu_{s}: L_{s} \times \prod_{i \in N} B_{i} \rightarrow \prod_{i \in N} B_{i}$. For each $s$ in $S$, let $X_{s}=L_{s} \times\left[p_{1}^{-1} \circ \alpha_{s}^{-1}\left(W_{s}+s\right) \cup p_{2}^{-1} \circ \alpha_{s}^{-1}\left(W_{s}+s\right)\right]$.

LEMMA 5. If $K \times \prod_{i \in N} B_{i}$ is uniformly isomorphic to $B^{\infty}$, then there is a collection $\left\{\chi_{s}\right\}_{s \in A}$ of homeomorphisms, $\chi_{s}: K \times \prod_{i \in N} B_{i} \rightarrow L_{s} \times \prod_{i \in N} B_{i}$, such that $\chi_{s} \mid X_{s}$ is the identity and the family $\left\{\chi_{s}\right\}_{s \in A} \cup\left\{\chi_{s}^{-1}\right\}_{s \in A}$ is uniformly equicontinuous.

Proof. Let $\mu_{K}$ be a uniform isomorphism of $K \times \prod_{i \in N} B_{i}$ onto $\prod_{i \in N} B_{i}$. It is easily seen that $\mu_{K}$ and each $\mu_{s}$ may be required to have the property that $p_{i} \circ p_{2}$ $=p_{i} \circ \mu_{\alpha}$, for $\alpha \in\{K\} \cup A$ and $i \in\{1,2\}$. This assumption gives, for each $s$ in $A$, $\mu_{K}\left(X_{s}\right) \subset \mu_{s}\left(X_{s}\right)=\bigcup_{i=1}^{2} p_{i}^{-1} \circ \alpha_{s}^{-1}\left(W_{s}+s\right)$.

It is a simple matter to construct a family $\left\{\xi_{s}\right\}_{s \in A}$ contained in $\mathscr{U}\left(\prod_{i \in N} B_{i}\right)$ such that $\left\{\xi_{s}\right\}_{s \in A} \cup\left\{\xi_{s}^{-1}\right\}_{s \in A}$ is uniformly equicontinuous and, for each $s$ in $A$, $\xi_{s}\left(\bigcup_{i=1}^{2} p_{i}^{-1} \circ \alpha_{s}^{-1}\left(W_{s}+s\right)\right)=p_{1}^{-1} \circ \alpha_{s}^{-1}\left(W_{s}+s\right)$. (One chooses a homeomorphism $\bar{\xi}$ of $[-1,1] \times[-1,1]$ onto itself such that $\bar{\xi}\left(\bigcup_{i=1}^{2} p_{i}^{-1}(1)\right)=p_{1}^{-1}(1)$ and defines $\xi_{s}$ to be $\left(\alpha_{s}^{-1} \times \alpha_{s}^{-1} \times \mathrm{id} \times \mathrm{id} \times \cdots\right) \circ\left(\xi_{1} \times \xi_{2} \times \mathrm{id} \times \mathrm{id} \times \cdots\right) \circ\left(\alpha_{s} \times \alpha_{s} \times \mathrm{id} \times \mathrm{id} \times \cdots\right)$, where $\xi_{i}=\left(I-f_{s}(\cdot) \cdot s\right) \circ p_{i}+\left[p_{i} \circ \bar{\xi} \circ\left(f_{s} \circ p_{1}, f_{s} \circ p_{2}\right)\right] \cdot s$, for $i=1$ or 2 . Now for each $s$ in $A$, let $H_{s}$ be given the norm under which $W_{s}$ is the unit ball (the Minkowski functional of $W_{s}$ ), and let $\lambda_{s}$ be the function given by Lemma 2 for the sequence $R, H_{s}, E_{2}, E_{3}, \ldots$ of Banach spaces. Let $\zeta_{s} \in \mathscr{U}\left(\prod_{\imath \in N} B_{i}\right)$ be

$$
\begin{aligned}
&\left(f_{s} \circ \alpha_{s} \circ p_{1},\left(I-f_{s}(\cdot) \cdot s\right) \circ \alpha_{s} \circ p_{1}, p_{2}, p_{3}, \ldots\right)^{-1} \circ \lambda_{s} \\
& \circ\left(f_{s} \circ \alpha_{s} \circ p_{1},\left(I-f_{s}(\cdot) \cdot s\right) \circ \alpha_{s} p_{1}, p_{2}, p_{3}, \ldots\right) .
\end{aligned}
$$

The family $\left\{\zeta_{s}\right\}_{s \in A} \cup\left\{\zeta_{s}^{-1}\right\}_{s \in A}$ is uniformly equicontinuous, and there is a collection $\left\{\delta_{i}\right\}_{i \in N} \subset(0,1)$ such that for $x$ in $p_{1}^{-1} \circ \alpha_{s}^{-1}\left(W_{s}+s\right), \zeta_{s}(x)$ is in $\alpha_{s}^{-1}\left(\delta_{1} W_{s}+s\right)$ $\times \prod_{i>1} \delta_{i} B_{i}$. Let $J=N \mid\{1\}$ and $M=\{2 i\}_{i \in N}$, and select, by Lemma 3, a uniform isomorphism $\varphi$ of $\prod_{i \in N} B_{i}$ onto itself such that $p_{1} \circ \varphi=p_{1}, p_{2 i} \circ \varphi\left(p_{1}^{-1}\left(S_{1}\right)\right)=0$ for all $i$ in $N$, and there is a collection $\left\{\varepsilon_{2 i+1}\right\}_{i \in N} \subset(0,1)$ for which $p_{2 i+1} \circ \varphi\left(p_{1}^{-1}\left(S_{1}\right)\right)$ $\subset \varepsilon_{2 i+1} \cdot B_{2 i+1}$ for all $i$ in $N$.

Let $\beta \in \mathscr{U}\left(\prod_{i \in N} B_{i}\right)$ be the function which exchanges the $2 i$ th- and $(2 i-1)$ thcoordinates for each $i$, and denote by $p_{0}$ and $p_{e}$ the projections of $\prod_{i \in N} B_{i}$ onto $\prod_{i \in N} B_{2 i-1}$ and $\prod_{i \in N} B_{2 i}$, respectively. The same symbols barred will be used to denote the projection followed by the injection into $\prod_{i \in N} B_{i}$ which inserts 0 in each of the other coordinates. Now let $\eta_{s}$ be the map from $\prod_{i \in N} B_{i}$ to $\prod_{i \in N} B_{i}$ given by

$$
\begin{aligned}
\eta_{s}= & \varphi \circ \zeta_{s} \circ \xi_{s} \circ \mu_{s} \circ \rho_{s} \circ \mu_{K}^{-1} \circ \xi_{s}^{-1} \circ \zeta_{s}^{-1} \circ \varphi^{-1} \circ \beta \circ \bar{p}_{e} \circ\left(\mathrm{id} \times \alpha_{s}^{-1} \times \mathrm{id} \times \mathrm{id} \times \cdots\right) \\
& \circ\left(\mathrm{id} \times\left(\mathrm{id}+s-f_{s}(\cdot) \cdot s\right) \times \mathrm{id} \times \mathrm{id} \times \cdots\right) \circ\left(\mathrm{id} \times \alpha_{s} \times \mathrm{id} \times \mathrm{id}, \ldots\right),
\end{aligned}
$$


where $\rho_{s}$ is the retraction of $K$ onto $L_{s}$ defined by first taking the second barycentric subdivision $K^{(2)}$ of $K$, then retracting $K^{(2)}$ onto $L_{s}^{(2)}$ by the simplicial map sending all vertices of $K^{(2)}$ not in $L_{s}^{(2)}$ to $v$ and then identifying $L_{s}^{(2)}$ with $L_{s}$. Let $\bar{\theta}_{s, 2 i-1}$ $=p_{2 i-1}+\left(1-\|\cdot\| \circ p_{2 i-1}\right) \cdot p_{2 i-1} \circ \eta_{s}$, if $i>1$, and let $\bar{\theta}_{s, 1}$ be

$$
\alpha_{s}^{-1} \circ\left[\alpha_{s} \circ p_{1}+\left(1-\|\cdot\|_{s} \circ\left(\mathrm{id}-f_{s}(\cdot) \cdot s\right) \circ \alpha_{s} \circ p_{1}\right) \cdot\left(\alpha_{s} \circ p_{1} \circ \eta_{s}(\cdot)-s\right)\right],
$$

where $\|\cdot\|_{s}$ is the norm indicated for $H_{s}$ under which $W_{s}$ is the unit ball. The function $\theta_{s}$ in $\mathscr{U}\left(\prod_{i \in N} B_{i}\right)$ given by $\theta_{s}=\left(\bar{\theta}_{s, 1}, p_{2}, \bar{\theta}_{s, 3}, p_{4}, \ldots\right)$ carries $\beta \circ \varphi \circ \zeta_{s}$ $\circ \xi_{s} \circ \mu_{K}\left(X_{s}\right)$ onto the graph of the function $p_{e} \circ \beta \circ \varphi \circ \zeta_{s} \circ \xi_{s} \circ \mu_{K} \circ \mu_{s}^{-1} \circ \xi_{s}^{-1} \circ \zeta_{s}^{-1}$ $\circ \varphi^{-1} \mid \varphi \circ \zeta_{s} \circ \xi_{s} \circ \mu_{s}\left(X_{s}\right)$ so that, for $x$ in $X_{s}, \theta_{s} \circ \beta \circ \varphi \circ \zeta_{s} \circ \xi_{s} \circ \mu_{K}(x)=\left(p_{0} \circ \varphi\right.$ $\left.\circ \zeta_{s} \circ \xi_{s} \circ \mu_{s}(x), p_{e} \circ \beta \circ \varphi \circ \zeta_{s} \circ \xi_{s} \circ \mu_{K}(x)\right)$. By a strictly analogous procedure, one may construct a family $\left\{\omega_{s}\right\}_{s \in A}$ of uniform isomorphisms of $\prod_{i \in N} B_{i}$ such that (1) $p_{0} \circ \omega_{s}=p_{0}$, (2) $\omega_{s}$ carries $\varphi \circ \zeta_{s} \circ \xi_{s} \circ \mu_{s}\left(X_{s}\right)$ onto $\theta_{s} \circ \beta \circ \varphi \circ \zeta_{s} \circ \xi_{s} \circ \mu_{K}\left(X_{s}\right)$, and (3) $\left\{\omega_{s}\right\}_{s \in A} \cup\left\{\omega_{s}^{-1}\right\}_{s \in A}$ is uniformly equicontinuous. Since $\left\{\theta_{s}\right\}_{s \in A} \cup\left\{\theta_{s}^{-1}\right\}_{s \in A}$ is uniformly equicontinuous, the desired family of uniform isomorphisms may be had by setting $\chi_{s}=\mu_{s}^{-1} \circ \xi_{s}^{-1} \circ \zeta_{s}^{-1} \circ \varphi^{-1} \circ \omega_{s}^{-1} \circ \theta_{s} \circ \beta \circ \varphi \circ \zeta_{s} \circ \xi_{s} \circ \mu_{K}$ for each $s$ in $A$. (This sort of extension procedure is due to V. L. Klee [13] in Hilbert spaces and has been elaborated upon by several authors, [1], [3], [8], and [18].)

Main theorems. In a metric space, a set $A$ will be called uniformly separated if there is a positive lower bound on the distances between pairs of points of $A$. The symbol $C(A)$ will denote the cardinality of $A$, and if $K$ is a simplicial complex, $c(K)$ will denote the least upper bound of $\left\{C\left(\mathrm{st}(v, K)^{\circ}\right) \mid v \in K^{\circ}\right\}$. The weight wt $(E)$ of a metric space $E$ is the greatest lower bound of the cardinalities of dense subsets of $E$. It is not difficult to show that when $E$ is an infinite-dimensional Hilbert space, then wt $(E)=\operatorname{dim}(E)$, and if $E$ is an infinite-dimensional Banach space, then it is of the same weight as its unit sphere. In fact, it is a theorem of A. H. Kruse [14] that in the latter case, there is a uniformly separated subset of the unit sphere of $E$ which has cardinality equal to wt $(E)$. Finally, defining a locally finite-dimensional simplicial complex to be one in which each point lies in a finitedimensional neighborhood, we have that this is equivalent to the requirement that the star of each vertex be finite dimensional.

THEOREM 3. Let $K$ be a metric, locally finite-dimensional, simplicial complex and $E$, a Banach space homeomorphic to its countably infinite Cartesian power. If $c(K) \leqq \mathrm{wt}(E)$, then $K \times E$ is an E-manifold.

Proof. With $B$ denoting the unit ball of $E$, it is sufficient to demonstrate that $K \times \prod_{i \in N} B_{i}$ is a $\prod_{i \in N} B_{i}$-manifold, for by a theorem of Bessaga and Klee [6] (see note added in proof), each infinite-dimensional Fréchet space is homeomorphic to each of its closed convex bodies. Thus, $E$ is homeomorphic to $B$ and to $\prod_{i \in N} E_{i}$, so it is homeomorphic to $\prod_{i \in N} B_{i}$. A second reduction of the problem is made by working with the vertex-stars of $K$, so it is sufficient to assume that $K$ is finite 
dimensional and the star of one of its vertices $v_{0}$. The proof proceeds by induction on the dimension of $K$.

The case that $\operatorname{dim}(K)=0$ is trivial, since then $K=\left\{v_{0}\right\}$. Assume then that $n \in N$ and that for any $(n-1)$-dimensional, metric, simplicial complex $L$ which is the star of one of its vertices, $L \times \prod_{i \in N} B_{i}$ is uniformly isomorphic to $\prod_{i \in N} B_{i}$ if the cardinality of $L^{\circ}$ is no greater than that of $K^{\circ}$. Now for any metric, $n$-dimensional simplicial complex $M$ which is the star of one of its vertices $v_{0}$ and which has no more vertices than $K$, there is a uniformly separated subset $A$ of the unit sphere of $B$ with the cardinality of the set of $n$-dimensional simplices of $M$. (This is because unless $K$ is $\left\{v_{0}\right\}, E$ must be infinite dimensional, in which case if $K$ has only finitely many vertices, the complex under consideration has at most finitely many $n$ simplices, and the unit sphere of $B$ is not totally bounded. Otherwise, if $K$ has infinitely many vertices, the cardinality of the $n$-simplices of $M$ cannot exceed that of the vertices of $K$.) By Lemma 4 there is a collection $\left\{\alpha_{s}\right\}_{s \in A}$ of uniform isomorphisms, $\alpha_{s}: B \rightarrow W_{s}+[-1,1] \cdot s$, where $W_{s}$ is a closed, symmetric, convex neighborhood of the origin in a codimension one hyperplane of $E$, with the property that there is a $\delta$ in $(0,1)$ such that $\alpha_{s}^{-1}\left(W_{s}+[1-\delta, 1] \cdot s\right) \subset(\varepsilon / 4) \cdot B+s$, where $\varepsilon$ in $(0,1)$ is chosen so that for $s$ and $t$ in $A,\|s-t\| \geqq \varepsilon$. Let the $n$-simplices of $M$ be indexed by $A$, and define $Z$ in $M \times \prod_{i \in N} B_{i}$ to be

$$
\left(\operatorname{st}\left(v_{0}, M^{n-1}\right) \times \prod_{i \in N} B_{i}\right) \cup \bigcup_{s \in A}\left(\Delta^{n}{ }_{s} \times \alpha_{s}^{-1}\left(W_{s}+s\right) \times \prod_{i>1} B_{i}\right) .
$$

The remainder of this proof consists of the showing that $Z$ is a uniform $\prod_{i \in N} B_{i^{-}}$ approximation to $M$.

For each $\zeta$ in $(0,1)$, construct a uniformly continuous retraction $f_{\zeta}$ of $M \times \prod_{i \in N} B_{i}$ onto $Z$ in the following manner: For each $s$ in $A$, let $\bar{f}_{\zeta, s}$ project $\Delta^{n}{ }_{s} \times\left(W_{s}+[-1,1] \cdot s\right)$ onto $\left(\Delta^{n}{ }_{s} \cap \operatorname{st}\left(v_{0}, M^{n-1}\right)\right) \times\left(W_{s}+[-1,1] \cdot s\right) \cup \Delta^{n}{ }_{s} \times\left(W_{s}+s\right)$ from the set $\left\{b_{s}\right\} \times\left[(-4 / \zeta) \cdot s+W_{s}\right]$ along the lines in

$$
\begin{aligned}
&\left\{\left[\left(b_{s}, y-(4 / \zeta) \cdot s\right),(x, y+t s)\right] \mid x \in \Delta^{n}{ }_{s} \cap s t\left(v_{0}, M^{n-1}\right), y \in W_{s}, t \in[-1,1]\right\} \\
& \cup\left\{\left[\left(b_{s}, y-(4 / \zeta) \cdot s\right),(x, y+s)\right] \mid x \in \Delta^{n}{ }_{s}, y \in W_{s}\right\},
\end{aligned}
$$

where $b_{s}$ is the barycenter of $\partial\left(\Delta^{n}\right) \mid s t\left(v_{0}, M^{n-1}\right)$. Let $f_{\zeta, s}=\left(\mathrm{id} \times\left(\alpha_{s}^{-1} \times \mathrm{id} \times \mathrm{id}\right.\right.$ $\times \cdots)) \circ\left(p_{1} \circ \bar{f}_{\zeta, s} \circ\left(p_{1}, p_{1} \circ p_{2}\right),\left(p_{2} \circ \bar{f}_{\zeta, s} \circ\left(p_{1}, p_{1} \circ p_{2}\right), p_{2} \circ p_{2}, p_{3} \circ p_{2}, \ldots\right)\right) \circ$ (id $\times\left(\alpha_{s} \times\right.$ id $\times$ id $\left.\left.\times \cdots\right)\right)$, and define

$$
\begin{aligned}
f_{\zeta}(x) & =f_{\zeta, s}(x) & & \text { if } p_{1}(x) \in \Delta^{n} \\
& =x & & \text { if } p_{1}(x) \in \text { st }\left(v_{0}, M^{n-1}\right) .
\end{aligned}
$$

This function is uniformly continuous because $\left\{\alpha_{s}\right\}_{s \in A} \cup\left\{\alpha_{s}^{-1}\right\}_{s \in A} \cup\left\{f_{\zeta, s}\right\}_{s \in A}$ is uniformly equicontinuous. Also, for any $x$ in $M, d\left(p_{1}(x), p_{1} \circ f_{\zeta}(x)\right) \leqq \zeta$.

Define $g_{\zeta}: M \times \prod_{i \in N} B_{i} \rightarrow\left(M \times \prod_{i \in N} B_{i}\right) \times\left(M \times \prod_{i \in N} B_{i}\right)$ to be $\left(f_{\zeta}\right.$, id $)$. Because $f_{\zeta}$ is uniformly continuous and $g_{\zeta}^{-1}=p_{2}, g_{\zeta}$ is a uniform embedding. Further, 
$d\left(p_{1} \circ p_{1} \circ g_{\zeta}(x), p_{1}(x)\right) \leqq \zeta$ for each $x$. Letting $Z_{i} \subset M \times \prod_{i \in N} B_{i} \times \prod_{i \in N} B_{i}$ be $\left(p_{1}, p_{i+1}\right)^{-1}(Z), i=1,2$, and

$$
\bar{g}_{\zeta}: M \times \prod_{i \in N} B_{i} \times \prod_{i \in N} B_{i} \rightarrow\left(M \times \prod_{i \in N} B_{i} \times \prod_{i \in N} B_{i}\right) \times\left(M \times \prod_{i \in N} B_{i}\right)
$$

be $\left(\left(p_{1} \circ p_{1} \circ g_{\zeta}, p_{2} \circ p_{1} \circ g_{\zeta}, p_{3}\right)\right.$, id), it is necessary to construct a uniform isomorphism $h_{\zeta}: \bar{g}_{\zeta}\left(Z_{2}\right) \rightarrow Z_{1}$ such that, for $x$ in $\bar{g}_{\zeta}\left(Z_{2}\right)$,

$$
d\left(\left(p_{1}, p_{2}\right) \circ h_{\zeta}(x),\left(p_{1} \circ p_{1}, p_{2} \circ p_{1}\right)(x)\right) \leqq \zeta .
$$

As $Z_{2}=\operatorname{st}\left(v_{0}, M^{n-1}\right) \times \prod_{i \in N} B_{i} \times \prod_{i \in N} B_{i} \cup \cup_{s \in A} \Delta_{s}^{n} \times \prod_{i \in N} B_{i} \times\left(\alpha_{s}^{-1}\left(W_{s}+s\right)\right.$ $\left.\times \prod_{i>1} B_{i}\right)$,

$$
\begin{aligned}
\bar{g}_{\zeta}\left(Z_{2}\right)= & \bar{g}_{\zeta}\left(\text { st }\left(v_{0}, M^{n-1}\right) \times \prod_{i \in N} B_{i} \times \prod_{i \in N} B_{i}\right) \\
& \cup \bigcup_{s \in A} \bar{g}_{\zeta}\left(\Delta^{n}{ }_{s} \times \prod_{i \in N} B_{i} \times\left(\alpha_{s}^{-1}\left(W_{s}+s\right) \times \prod_{i>1} B_{i}\right)\right) .
\end{aligned}
$$

The set

$$
\begin{aligned}
\bar{g}_{\zeta}\left(\operatorname{st}\left(v_{0}, M^{n-1}\right)\right. & \left.\times \prod_{i \in N} B_{i} \times \prod_{i \in N} B_{i}\right) \\
= & \left\{(x, y, z, x, y) \mid(x, y, z) \in \operatorname{st}\left(v_{0}, M^{n-1}\right) \times \prod_{i \in N} B_{i} \times \prod_{i \in N} B_{i}\right\} .
\end{aligned}
$$

The set $\bar{g}_{\zeta}\left(\Delta^{n}{ }_{s} \times \prod_{i \in N} B_{i} \times\left(\alpha_{s}^{-1}\left(W_{s}+s\right) \times \prod_{i>1} B_{i}\right)\right)=C_{s} \cup D_{s}$, where

$C_{s}=\left\{\left(x, y, z, x^{\prime}, y^{\prime}\right) \mid x \in \Delta_{s}^{n} ; y, z \in \alpha_{s}^{-1}\left(W_{s}+s\right) \times \prod_{i>1} B_{i} ;\right.$

$$
\begin{array}{r}
p_{i}(y)=p_{i}\left(y^{\prime}\right) \text { for } i>1 ; \alpha_{s} \circ p_{1}(y)-\alpha_{s} \circ p_{1}\left(y^{\prime}\right) \in[0,2] \cdot s \\
\left.\left(x^{\prime}, \alpha_{s} \circ p_{1}\left(y^{\prime}\right)\right) \in\left[\left(b_{s},(-4 / \zeta-1) s+\alpha_{s} \circ p_{1}(y)\right),\left(x, \alpha_{s}(y)\right)\right]\right\}
\end{array}
$$

and

$$
\begin{aligned}
& D_{s}=\left\{\left(x, y, z, x^{\prime}, y^{\prime}\right) \mid\right. x \in \Delta_{s}^{n} \cap \text { st }\left(v_{0}, M^{n-1}\right) ; z \in \alpha_{s}^{-1}\left(W_{s}+s\right) \times \prod_{i>1} B_{i} \\
& y \in \prod_{i \in n} B_{i} ; p_{i}(y)=p_{i}\left(y^{\prime}\right) \text { for } i>1 \\
& \alpha_{s} \circ p_{1}(y)-\alpha_{s} \circ p_{1}\left(y^{\prime}\right) \in\left[0,1+f_{s} \circ \alpha_{s} \circ p_{1}(y)\right] \cdot s \text { and } \\
&\left(x^{\prime}, \alpha_{s} \circ p_{1}\left(y^{\prime}\right)\right)\left.\in\left[\left(b_{s}, \alpha_{s} \circ p_{1}(y)-\left(f_{s} \circ \alpha_{s} \circ p_{1}(y)+4 / \zeta\right) \cdot s\right),\left(x, \alpha_{s} \circ p_{1}(y)\right)\right]\right\} \cdot
\end{aligned}
$$

(Here, $f_{s}$ is the same functional as used in the proof of Lemma 4 to construct $\alpha_{s}$ and $\left.W_{s .}\right)$ Therefore, $p=\left(p_{1}, p_{2} \circ p_{2}\right) \mid \bar{g}_{\zeta}\left(Z_{2}\right): \bar{g}_{\zeta}\left(Z_{2}\right) \rightarrow\left(M \times \prod_{i \in N} B_{i} \times \prod_{i \in N} B_{i}\right) \times \prod_{i \in N} B_{i}$ is a uniform embedding. (It is clearly uniformly continuous. It is one-to-one because if $(x, y, z)$ and $\left(x^{\prime}, y, z^{\prime}\right)$ are distinct points of $Z_{2}$ such that $f_{\xi}(x, y)=f_{\xi}\left(x^{\prime}, y\right)$, then there must be two distinct elements $s$ and $s^{\prime}$ of $A$ such that $x \in \Delta^{n}{ }_{s} \mid s t\left(v_{0}, M^{n-1}\right)$ and $x^{\prime} \in \Delta_{s^{\prime}}^{n} \mid$ st $\left(v_{0}, M^{n-1}\right)$, since $f_{\zeta}(a, b) \neq(a, b)$ implies $p_{2} \circ f_{\zeta}(a, b) \neq b$. Then, 
however, $p_{1}(z) \in \alpha_{s}^{-1}\left(W_{s}+s\right)$ and $p_{1}\left(z^{\prime}\right) \in \alpha_{s^{\prime}}{ }^{-1}\left(W_{s^{\prime}}+s^{\prime}\right)$, which are disjoint by the choice of $\delta$. The uniform continuity of $p^{-1}$ may be seen by observing that on $p \circ \bar{g}_{\zeta}\left(\mathrm{st}\left(v_{0}, M^{n-1}\right) \times \prod_{i \in N} B_{i} \times \prod_{i \in N} B_{i}\right), \quad p^{-1}=\left(p_{1},\left(p_{1} \circ p_{1}, p_{2}\right)\right)$ and that on $p\left(C_{s} \cup D_{s}\right), p^{-1}=\left(p_{1},\left(\tau_{s}(\cdot) \cdot b_{s}+\left(1-\tau_{s}(\cdot)\right) \cdot p_{1} \circ p_{1}, p_{2}\right)\right)$, where

$$
\tau_{s}=\left(f_{s} \circ \alpha_{s} \circ p_{1} \circ p_{2} \circ p_{1}-f_{s} \circ \alpha_{s} \circ p_{1} \circ p_{2}\right) /\left(4 / \zeta+f_{s} \circ \alpha_{s} \circ p_{1} \circ p_{2} \circ p_{1}\right) .
$$

Because $\left\{\tau_{s}\right\}_{s \in A}$ is uniformly equicontinuous and $\left\{p\left(C_{s} \cup D_{s}\right)\right\}_{s \in A}$ is composed of sets which are uniformly separated from each other, $p^{-1}$ is uniformly continuous if it is continuous. It is continuous because for

$$
\left(x, y, z, y^{\prime}\right) \in p \circ \bar{g}_{\zeta}\left(\operatorname{st}\left(v_{0}, M^{n-1}\right) \times \prod_{i \in N} B_{i} \times \prod_{i \in N} B_{i}\right) \cap p\left(C_{s} \cup D_{s}\right),
$$

$y=y^{\prime}$ and, thus, $\tau_{s}\left(x, y, z, y^{\prime}\right)=0$.)

The structure of $p \circ \bar{g}_{\zeta}\left(Z_{2}\right)$ is as follows:

(1) $p \circ \bar{g}_{\zeta}\left(\operatorname{st}\left(v_{0}, M^{n-1}\right) \times \prod_{i \in N} B_{i} \times \prod_{i \in N} B_{i}\right)=\left\{(x, y, z, y) \mid(x, y, z) \in \operatorname{st}\left(v_{0}, M^{n-1}\right)\right.$ $\left.\times \prod_{i \in N} B_{i} \times \prod_{i \in N} B_{i}\right\}$,

(2) $p\left(C_{s}\right)=\left\{\left(x, y, z, y^{\prime}\right) \mid x \in \Delta^{n} ; y, z \in \alpha_{s}^{-1}\left(W_{s}+s\right) \times \prod_{i>1} B_{i} ; p_{i}(y)=p_{i}\left(y^{\prime}\right)\right.$ if $\left.i>1 ; \alpha_{s} \circ p_{1}(y)-\alpha_{s} \circ p_{1}\left(y^{\prime}\right) \in[0,2] \cdot s\right\}$, and

(3) $p\left(D_{s}\right)=\left\{\left(x, y, z, y^{\prime}\right) \mid x \in \Delta_{s}^{n} \cap\right.$ st $\left(v_{0}, M^{n-1}\right) ; z \in \alpha_{s}^{-1}\left(W_{s}+s\right) \times \prod_{i>1} B_{i}$; $p_{i}(y)=p_{i}\left(y^{\prime}\right)$ for $i>1$, and $\alpha_{s} \circ p_{1}\left(y^{\prime}\right) \in \alpha_{s} \circ p_{1}(y)-f_{s} \circ \alpha_{s} \circ p_{1}(y) \cdot s+\left[-1-f_{s} \circ \alpha_{s}\right.$ $\left.\left.\circ p_{1}(y), 0\right] \cdot s\right\}$.

Let $\beta: p \circ \bar{g}_{\zeta}\left(Z_{2}\right) \rightarrow\left(M \times \prod_{i \in N} B_{i} \times \prod_{i \in N} B_{i}\right) \times[0,2]$ be defined by

$$
\beta \mid\left(p_{1} \circ p_{1}\right)^{-1}\left(\Delta_{s}^{n}\right)=\left(p_{1},\|\cdot\| \circ\left(\alpha_{s} \circ p_{1} \circ p_{2}-\alpha_{s} \circ p_{1} \circ p_{2} \circ p_{1}\right)\right)
$$

for each $s$ in $A$ and $\beta \mid\left(p_{1} \circ p_{1}\right)^{-1}$ (st $\left.\left(v_{0}, M^{n-1}\right)\right)=\left(p_{1}, 0\right)$. (This is also a uniform isomorphism, for on $\bigcup_{s \in A} p\left(C_{s} \cup D_{s}\right)$ it is $\bigcup_{s \in A}\left(p_{1},\|\cdot\| \circ\left(\alpha_{s} \circ p_{1} \circ p_{2}-\alpha_{s} \circ p_{1} \circ p_{2} \circ p_{1}\right)\right)$, the sets $p\left(C_{s} \cup D_{s}\right)$ are uniformly separated, and on

$$
p \circ \bar{g}_{\zeta}\left(\operatorname{st}\left(v_{0}, M^{n-1}\right) \times \prod_{i \in N} B_{i} \times \prod_{i \in N} B_{i}\right) \cap p\left(C_{s} \cup D_{s}\right),
$$

the function $p_{2} \circ p_{1}$ agrees with $p_{2}$, which shows continuity, hence uniform continuity, of the inverse.)

An examination yields that

$$
\beta \circ p \circ \bar{g}_{\zeta}\left(\operatorname{st}\left(v_{0}, M^{n-1}\right) \times \prod_{i \in N} B_{i} \times \prod_{i \in N} B_{i}\right)=\left(\operatorname{st}\left(v_{0}, M^{n-1}\right) \times \prod_{i \in N} B_{i} \times \prod_{i \in N} B_{i}\right) \times\{0\},
$$

that for each $s$ in $A, \beta \circ p\left(C_{s}\right)=\left[\Delta^{n}{ }_{s} \times\left(\alpha_{s}^{-1}\left(W_{s}+s\right) \times \prod_{i>1} B_{i}\right) \times\left(\alpha_{s}^{-1}\left(W_{s}+s\right)\right.\right.$ $\left.\left.\times \prod_{i>1} B_{i}\right)\right] \times[0,2]$, and that $\beta \circ p\left(D_{s}\right)=\left\{(x, y, z, t) \mid x \in \Delta^{n}{ }_{s} \cap \mathrm{st}\left(v_{0}, M^{n-1}\right)\right.$; $y \in \prod_{i \in N} B_{i} ; z \in \alpha_{s}^{-1}\left(W_{s}+s\right) \times \prod_{i>1} B_{i}$, and $\left.0 \leqq t \leqq 1+f_{s} \circ \alpha_{s} \circ p_{1}(y)\right\}$.

Let $\eta(\zeta) \in(0, \delta)$ be small enough that for any $s$ in $A$ and any $x$ and $y$ in $W_{s}$ $+[-1,1] \cdot s$ with $\|x-y\| \leqq \eta(\zeta),\left\|\alpha_{s}^{-1}(x)-\alpha_{s}^{-1}(y)\right\| \leqq \zeta / 3$, and let

$$
\bar{\gamma}_{\zeta}:\{(s, t) \in[-1,1] \times[0,2] \mid t \leqq 1+s\} \rightarrow[-1,1] \times[0,2]
$$


be any homeomorphism which is the identity on $[-1,1] \times\{0\} \cup\{1\} \times[0,2]$ and changes no point's first coordinate more than $\eta(\zeta)$. Let $\gamma_{\zeta}: \beta \circ p \circ g_{\zeta}\left(Z_{2}\right)$ $\rightarrow\left(M \times \prod_{i \in N} B_{i} \times \prod_{i \in N} B_{i}\right) \times[0,2]$ be $\left(\left(p_{1} \circ p_{1},\left(\alpha_{s}{ }^{-1} \circ\left(\left(\alpha_{s}-f_{s} \circ \alpha_{s}(\cdot)\right) \cdot s\right) \circ p_{1} \circ p_{2}\right.\right.\right.$ $\left.\left.\circ p_{1}+\left(p_{1} \circ \bar{\gamma}_{\zeta} \circ\left(f_{s} \circ \alpha_{s} \circ p_{1} \circ p_{2} \circ p_{1}, p_{2}\right) \cdot s\right), p_{2} \circ p_{2} \circ p_{1}, p_{3} \circ p_{2} \circ p_{1}, \ldots\right), p_{3} \circ p_{1}\right)$, $\left.p_{2} \circ \bar{\gamma}_{\zeta} \circ\left(f_{s} \circ \alpha_{s} \circ p_{1} \circ p_{2} \circ p_{1}, p_{2}\right)\right)$ on $\left(\left(p_{1} \circ p_{1}\right), p_{1} \circ p_{3} \circ p_{1}\right)^{-1}\left(\Delta^{n}{ }_{s} \times \alpha_{s}{ }^{-1}\left(W_{s}+s\right)\right)$ and the identity map on $p_{2}^{-1}(0)$. That $\gamma_{\zeta}$ is a uniform isomorphism follows in a similar way to the argument for $\beta$. That for any point $x$ in $\beta \circ p \circ \bar{g}_{\xi}\left(Z_{2}\right)$, $d\left(\left(p_{1} \circ p_{1}, p_{2} \circ p_{1}\right) \circ \gamma_{\zeta}(x),\left(p_{1} \circ p_{1}, p_{2}, \circ p_{1}\right)(x)\right)=d\left(p_{1} \circ p_{2} \circ p_{1} \circ \gamma_{\zeta}(x), p_{1} \circ p_{2} \circ p_{1}(x)\right)$ $\leqq \zeta / 3$ is given by the choice of $\eta(\zeta)$ and the construction of $\bar{\gamma}_{\zeta}$.

Upon inspection and reference to the second paragraph back it is immediate that

$$
\begin{aligned}
\gamma_{\zeta} \circ \beta \circ p \circ \bar{g}_{\zeta}\left(Z_{2}\right)=\left(\operatorname{st}\left(v_{0}, M^{n-1}\right) \times \prod_{i \in N} B_{i} \times \prod_{i \in N} B_{i}\right) \times\{0\} \\
\cup \bigcup_{s \in A}\left[\left(\Delta^{n} \cap \cap \operatorname{st}\left(v_{0}, M^{n-1}\right)\right) \times \prod_{i \in N} B_{i} \times\left(\alpha_{s}{ }^{-1}\left(W_{s}+s\right) \times \prod_{i>1} B_{i}\right)\right] \times[0,2] .
\end{aligned}
$$

For each $s$ in $A$, let $\bar{\mu}_{s}$ be a homeomorphism of $\Delta^{n}{ }_{s} \cap$ st $\left(v_{0}, M^{n-1}\right)$ onto $[-1,1]^{n-1}$ in such a manner that $\{\bar{\mu}\}_{s \in A} \cup\left\{\bar{\mu}_{s}^{-1}\right\}_{s \in A}$ is uniformly equicontinuous. Select $s_{0}$ in $A$ and let $\mu:[-1,1]^{n-1} \times \prod_{i \in N} B_{i} \rightarrow \prod_{i \in N} B_{i}$ be the function $\left(\mathrm{id} \times \mathrm{id} \times \alpha_{s_{0}}^{-1} \times \alpha_{s_{0}}^{-1} \times \cdots\right) \circ\left(p_{1} \circ p_{2}, p_{2} \circ p_{2},\left(\mathrm{id}-f_{s}(\cdot) \cdot s\right) \circ p_{3} \circ p_{2}+\left(p_{1} \circ p_{1}(\cdot)\right) \cdot s\right.$, $\ldots,\left(\mathrm{id}-f_{s}(\cdot) \cdot s\right) \circ p_{n+1} \circ p_{2}+\left(p_{n-1} \circ p_{1}(\cdot)\right) \cdot s,\left(\mathrm{id}-f_{s}(\cdot) \cdot s\right) \circ p_{n+2} \circ p_{2}+\left(f_{s} \circ p_{3}\right.$ $\left.\left.\circ p_{2}(\cdot)\right) \cdot s, \ldots,\left(\mathrm{id}-f_{s}(\cdot) \cdot s\right) \circ p_{m+n-1} \circ p_{2}+\left(f_{s} \circ p_{m} \circ p_{2}(\cdot)\right) \cdot s, \ldots\right) \circ(\mathrm{id} \times(\mathrm{id} \times \mathrm{id}$ $\left.\left.\times \alpha_{s_{0}} \times \alpha_{s_{0}} \times \cdots\right)\right)$. For each $s$ in $A$, let $\mu_{s}: \Delta^{n}{ }_{s} \cap$ st $\left(v_{0}, M^{n-1}\right) \times \prod_{i \in N} B_{i} \times \prod_{i \in N} B_{i}$ $\rightarrow \prod_{i \in N} B_{i}$ be

$$
\mu \circ\left(\bar{\mu}_{s} \circ p_{1},\left(p_{1} \circ p_{2}, p_{1} \circ p_{3}, p_{2} \circ p_{2}, p_{2} \circ p_{3}, \ldots, p_{n} \circ p_{2}, p_{n} \circ p_{3}, \ldots\right)\right) \text {. }
$$

By hypothesis, st $\left(v_{0}, M^{n-1}\right) \times \prod_{i \in N} B_{i} \times \prod_{i \in N} B_{i}$ is uniformly isomorphic to $\prod_{i \in N} B_{i}$, so by Lemma 5 there is a family $\left\{\bar{\chi}_{s}\right\}_{s \in A}$ of uniform isomorphisms $\bar{\chi}_{s}$ : st $\left(v_{0}, M^{n-1}\right) \times \prod_{i \in N} B_{i} \times \prod_{i \in N} B_{i} \rightarrow\left(\Delta^{n}{ }_{s} \cap\right.$ st $\left.\left(v_{0}, M^{n-1}\right)\right) \times \prod_{i \in N} B_{i} \times \prod_{i \in N} B_{i}$ such that $\left\{\bar{\chi}_{s}\right\}_{s \in A} \cup\left\{\bar{\chi}_{s}^{-1}\right\}_{s \in A}$ is uniformly equicontinuous and, for each $s$, $\bar{\chi}_{s} \mid \cup_{i=1}^{2}\left(p_{1}, p_{1} \circ p_{i+1}\right)^{-1}\left(\left[\Delta^{n}{ }_{s} \cap s t\left(v_{0}, M^{n-1}\right)\right] \times \alpha_{s}{ }^{-1}\left(W_{s}+s\right)\right)$ is the identity. Each $\bar{\chi}_{s}$ defines a uniform isomorphism, which will be called $\chi_{s}$, of

$$
\begin{aligned}
\left(\operatorname{st}\left(v_{0}, M^{n-1}\right) \times \prod_{i \in N} B_{i} \times \prod_{i \in N} B_{i}\right) \times\{0\} \\
\cup\left[\left(\Delta^{n}{ }_{s} \cap \operatorname{st}\left(v_{0}, M^{n-1}\right)\right) \times \prod_{i \in N} B_{i} \times\left(\alpha_{s}{ }^{-1}\left(W_{s}+s\right) \times \prod_{i>1} B_{i}\right)\right] \times[0,2]
\end{aligned}
$$

onto

$$
\begin{aligned}
& {\left[\left(\Delta^{n}{ }_{s} \cap \text { st }\left(v_{0}, M^{n-1}\right)\right) \times \prod_{i \in N} B_{i} \times \prod_{i \in N} B_{i}\right] \times\{0\}} \\
& \quad \cup\left[\left(\Delta^{n}{ }_{s} \cap \operatorname{st}\left(v_{0}, M^{n-1}\right)\right) \times \prod_{i \in N} B_{i} \times\left(\alpha_{s}^{-1}\left(W_{s}+s\right) \times \prod_{i>1} B_{i}\right)\right] \times[0,2]
\end{aligned}
$$

which is given by $\chi_{s}=\bar{\chi}_{s} \times$ id. 
Let $\bar{\eta}(\zeta) \in(0, \min \{\varepsilon / 8, \zeta / 20\})$ be small enough that for each $s$ in $A$ and each pair, $x$ and $y$, of points in the domain of $\chi_{s}^{-1}$ with $d(x, y) \leqq \bar{\eta}(\zeta), d\left(\chi_{s}^{-1}(x), \chi_{s}^{-1}(y)\right)$ $\leqq \min \{\varepsilon / 8, \zeta / 20\}$, and let $\delta(\zeta) \in(0, \delta)$ be small enough that for each $s$ in $A$ and each pair $x$ and $y$, of points of $W_{s}+[-1,1] \cdot s$ with $\|x-y\| \leqq \delta(\zeta),\left\|\alpha_{s}{ }^{-1}(x)-\alpha_{s}{ }^{-1}(y)\right\|$ $\leqq \bar{\eta}(\zeta)$. Now let $\omega_{\zeta}:[-1,1] \times\{0\} \cup\{1\} \times[0,2] \rightarrow[-1,1] \times\{0\}$ be a homeomorphism which is the identity on $[-1,1-\delta(\zeta)] \times\{0\}$, and for each $s$ in $A$ let

$$
\begin{aligned}
\omega_{s, \zeta}:\left[\left(\Delta^{n} \cap\right.\right. \text { st } & \left.\left.\left(v_{0}, M^{n-1}\right)\right) \times \prod_{i \in N} B_{i} \times \prod_{i \in N} B_{i}\right] \times\{0\} \\
& \cup\left[\left(\Delta^{n}{ }_{s} \cap \operatorname{st}\left(v_{0}, M^{n-1}\right)\right) \times \prod_{i \in N} B_{i} \times\left(\alpha_{s}{ }^{-1}\left(W_{s}+s\right) \times \prod_{i>1} B_{i}\right)\right] \times[0,2] \\
\rightarrow & {\left[\left(\Delta^{n}{ }_{s} \cap \operatorname{st}\left(v_{0}, M^{n-1}\right)\right) \times \prod_{i \in N} B_{i} \times \prod_{i \in N} B_{i}\right] \times\{0\} }
\end{aligned}
$$

be $\left(\left(\mathrm{id} \times \mathrm{id} \times\left(\alpha_{s}{ }^{-1} \times \mathrm{id} \times \mathrm{id} \times \cdots\right)\right) \times \mathrm{id}\right) \circ\left(\left(p_{1} \circ p_{1}, p_{2} \circ p_{1},\left(p_{1} \circ p_{3} \circ p_{1}-\left(f_{s} \circ p_{1} \circ p_{3}\right.\right.\right.\right.$ $\left.\left.\left.\left.\circ p_{1}(\cdot)\right) \cdot s+p_{1} \circ \omega_{\zeta} \circ\left(f_{s} \circ p_{1} \circ p_{3} \circ p_{1}, p_{2}\right), p_{2} \circ p_{3} \circ p_{1}, p_{3} \circ p_{3} \circ p_{1}, \ldots\right)\right), 0\right) \circ((\mathrm{id} \times \mathrm{id}$ $\left.\left.\times\left(\alpha_{s} \times \mathrm{id} \times \mathrm{id} \times \cdots\right)\right) \times \mathrm{id}\right)$.

Let $\psi_{\zeta}:[-1,1] \times[-1,1] \rightarrow[-1,1] \times[-1,1]$ be a homeomorphism which is the identity on $[-1,1-\delta(\zeta)] \times[-1,1]$ and carries $\{1\} \times p_{1} \circ \omega_{\zeta}(\{1\} \times[0,2])$ onto $\{1\} \times[-1,1]$. For each $s$ in $A$, let $\psi_{s, \zeta}$ be the uniform isomorphism of $\left[\left(\Delta^{n}{ }_{s} \cap \mathrm{st}\left(v_{0}, M^{n-1}\right)\right) \times \prod_{i \in N} B_{i} \times \prod_{i \in N} B_{i}\right] \times\{0\}$ onto itself given by $\psi_{s, \zeta}=((\mathrm{id}$ $\left.\left.\times\left(\alpha_{s}{ }^{-1} \times \mathrm{id} \times \cdots\right) \times\left(\alpha_{s}{ }^{-1} \times \mathrm{id} \times \cdots\right)\right) \times \mathrm{id}\right) \circ\left(\left(p_{1} \circ p_{1},\left(p_{1} \circ p_{2} \circ p_{1}-\left(f_{s} \circ p_{1} \circ p_{2}\right.\right.\right.\right.$ $\left.\left.\circ p_{1}(\cdot)\right) \cdot s+p_{1} \circ \psi_{\zeta} \circ\left(f_{s} \circ p_{1} \circ p_{2} \circ p_{1}, f_{s} \circ p_{1} \circ p_{3} \circ p_{1}\right), p_{2} \circ p_{2} \circ p_{1}, p_{3} \circ p_{2} \circ p_{1}, \ldots\right)$, $\left(p_{1} \circ p_{3} \circ p_{1}-\left(f_{s} \circ p_{1} \circ p_{3} \circ p_{1}(\cdot)\right) \cdot s+p_{2} \circ \psi_{\zeta} \circ\left(f_{s} \circ p_{1} \circ p_{2} \circ p_{1}, f_{s} \circ p_{1} \circ p_{3} \circ p_{1}\right), p_{2}\right.$ $\left.\left.\left.\circ p_{3} \circ p_{1}, \ldots\right)\right), p_{2}^{*}\right) \circ\left(\left(\mathrm{id} \times\left(\alpha_{s} \times \mathrm{id} \times \cdots\right) \times\left(\alpha_{s} \times \mathrm{id} \times \cdots\right)\right) \times \mathrm{id}\right)$.

For each $s$ in $A$ let

$$
\begin{aligned}
& \bar{\xi}_{s, \zeta}: {\left[\left(\Delta^{n} \cap \text { st }\left(v_{0}, M^{n-1}\right)\right) \times \prod_{i \in N} B_{i} \times\left(\alpha_{s}^{-1}\left(W_{s}+s\right) \times \prod_{i>1} B_{i}\right)\right] \times[0,2] } \\
& \cup\left(\operatorname{st}\left(v_{0}, M^{n-1}\right) \times \prod_{i \in N} B_{i} \times \prod_{i \in N} B_{i}\right) \times\{0\} \rightarrow\left(\text { st }\left(v_{0}, M^{n-1}\right) \times \prod_{i \in N} B_{i} \times \prod_{i \in N} B_{i}\right) \times\{0\}
\end{aligned}
$$

be $\chi_{s}{ }^{-1} \circ \omega_{s, \zeta} \circ \chi_{s}$. The $\xi_{s, \zeta}$ 's are supported on mutually disjoint subsets of

$$
\begin{aligned}
& \left(\text { st }\left(v_{0}, M^{n-1}\right) \times \prod_{i \in N} B_{i} \times \prod_{i \in N} B_{i}\right) \times\{0\} \\
& \cup \bigcup_{s \in A}\left[\left(\Delta^{n}{ }_{s} \cap \operatorname{st}\left(v_{0}, M^{n-1}\right)\right) \times \prod_{i \in N} B_{i} \times\left(\alpha_{s}{ }^{-1}\left(W_{s}+s\right) \times \prod_{i>1} B_{i}\right)\right] \times[0,2] .
\end{aligned}
$$

(This is because $\omega_{s, \zeta}(x) \neq x$ implies that $\omega_{s, \zeta}(x)$ is in $\left[\left(\Delta^{n}{ }_{s} \cap s t\left(v_{0}, M^{n-1}\right)\right)\right.$ $\left.\times \prod_{i \in N} B_{i} \times\left(\alpha_{s}^{-1}\left(W_{s}+[1-\delta(\zeta), 1] \cdot s\right) \times \prod_{i>1} B_{i}\right)\right] \times\{0\}$, which by the choice of $\delta(\zeta)$ is within the open $(\varepsilon / 8)$-neighborhood of $\left(p_{1} \circ p_{3} \circ p_{1}\right)^{-1}(s)$-because of the scaling-down of the metric in accordance with the convention made in the proof of Theorem 2. Also, by the choice of $\delta(\zeta)$, there is a point $y$ of $\left(p_{1} \circ p_{3} \circ p_{1}\right)^{-1}(s)$ within $\bar{\eta}(\zeta)$ of $\omega_{s, \zeta}(x)$. This gives $d\left(\chi_{s}^{-1}(y), \chi_{s}^{-1}(x)\right) \leqq \min \{\varepsilon / 8, \zeta / 20\}$, so $\chi_{s}{ }^{-1} \circ \omega_{s}(x)$ 
is within $\varepsilon / 4$ of some point of $\left(p_{1} \circ p_{3} \circ p_{1}\right)^{-1}(s)$. This establishes that the supports of the $\xi_{s, \zeta}$ 's are disjoint, for the distance between any point of $\left(p_{1} \circ p_{3} \circ p_{1}\right)^{-1}(s)$ and any point of $\left(p_{1} \circ p_{3} \circ p_{1}\right)^{-1}(A \backslash\{s\})$ is at least $\varepsilon / 2$.) Therefore, there exists a function

$$
\begin{aligned}
\xi_{\zeta}:\left(\operatorname{st}\left(v_{0}, M^{n-1}\right) \times \prod_{i \in N} B_{i} \times \prod_{i \in N} B_{i}\right) \times\{0\} \\
\cup \bigcup_{s \in A}\left[\left(\Delta^{n}{ }_{s} \cap \operatorname{st}\left(v_{0}, M^{n-1}\right)\right) \times \prod_{i \in N} B_{i} \times\left(\alpha_{s}{ }^{-1}\left(W_{s}+s\right) \times \prod_{i>1} B_{i}\right)\right] \times[0,2] \\
\rightarrow\left(\operatorname{st}\left(v_{0}, M^{n-1}\right) \times \prod_{i \in N} B_{i} \times \prod_{i \in N} B_{i}\right) \times\{0\}
\end{aligned}
$$

defined by the formula

$$
\begin{aligned}
\xi_{\zeta}(x) & =\xi_{s, \zeta}(x) & & \text { if } \xi_{s, \zeta}(x) \neq x, \\
& =x & & \text { if } \xi_{s, \zeta}(x)=x \text { for all } s \text { in } A .
\end{aligned}
$$

The uniform equicontinuity of the family $\left\{\xi_{s, \zeta}\right\}_{s \in A} \cup\left\{\xi_{s, \zeta}{ }^{-1}\right\}_{s \in A}$ assures that $\xi_{\zeta}$ is a uniform isomorphism. Observe that by the choice of $\delta(\zeta)$ and $\bar{\eta}(\zeta)$,

$$
d\left(\left(p_{1} \circ p_{1}, p_{2} \circ p_{1}\right) \circ \xi_{\zeta}(x),\left(p_{1} \circ p_{1}, p_{2} \circ p_{1}\right)(x)\right) \leqq \zeta / 20
$$

for each $x$ in the domain of $\xi_{\zeta}$.

Utilizing the functions $\left\{\psi_{s, \zeta}\right\}_{s \in A}$, let $\theta_{s, \zeta}=\chi_{s}{ }^{-1} \circ \psi_{s, \zeta} \circ \chi_{s}$. The supports of the $\theta_{s, \zeta}$ 's are also mutually disjoint, and there is defined a uniform isomorphism $\theta_{\zeta}$ of (st $\left.\left(v_{0}, M^{n-1}\right) \times \prod_{i \in N} B_{i} \times \prod_{i \in N} B_{i}\right) \times\{0\}$ onto itself which has the property that for $x$ in $\left(\right.$ st $\left.\left(v_{0}, M^{n-1}\right) \times \prod_{i \in N} B_{i} \times \prod_{i \in N} B_{i}\right) \times\{0\}$,

$$
d\left(\left(p_{1} \circ p_{1}, p_{2} \circ p_{1}\right) \circ \theta_{\zeta}(x),\left(p_{1} \circ p_{1}, p_{2} \circ p_{1}\right)(x)\right) \leqq \zeta / 4 \text {. }
$$

(This is because if $\theta_{\zeta}(x) \neq x$, there is an $s$ in $A$ for which $\theta_{\zeta}(x)=\theta_{s, \zeta}(x)=\chi_{s}{ }^{-1} \circ \psi_{s, \zeta}$ $\circ \chi_{s}(x)$. Since $\psi_{s, \zeta} \circ \chi_{s}(x) \neq \chi_{s}(x), \chi_{s}(x)$ must be within $\bar{\eta}(\zeta)$ of some point $y$ in the fixed point set of $\chi_{s}$, so $d\left(x, \chi_{s}(x)\right) \leqq \bar{\eta}(\zeta)+\zeta / 20 \leqq \zeta / 10$. Also,

$$
d\left(\left(p_{1} \circ p_{1}, p_{2} \circ p_{1}\right)\left(\chi_{s}(x)\right),\left(p_{1} \circ p_{1}, p_{2} \circ p_{1}\right) \circ \psi_{s, \zeta} \circ \chi_{s}(x)\right) \leqq \bar{\eta}(\zeta) \leqq \zeta / 20,
$$

and $\psi_{s, \zeta} \circ \chi_{s}(x)$ must also lie within $\bar{\eta}(\zeta)$ of some point $z$ of the fixed-point set of $\chi_{s}$. Thus, $d\left(\psi_{s, \zeta} \circ \chi_{s}(x), \theta_{s, \zeta}(x)\right) \leqq \zeta / 10$. Combining the inequalities gives the result.)

Consider now, for $s$ in $A$,

$$
\begin{aligned}
\theta_{s, \zeta} \circ \xi_{s, \zeta}\left(\left[\left(\Delta^{n} \cap \operatorname{st}\left(v_{0}, M^{n-1}\right)\right) \times\left(\alpha_{s}^{-1}\left(W_{s}+s\right)\right.\right.\right. & \left.\times \prod_{i>1} B_{i}\right) \\
& \left.\left.\times\left(\alpha_{s}^{-1}\left(W_{s}+s\right) \times \prod_{i>1} B_{i}\right)\right] \times[0,2]\right) .
\end{aligned}
$$

It is $\left.\left[\left(\Delta^{n}{ }_{s} \cap \operatorname{st}\left(v_{0}, M^{n-1}\right)\right) \times\left(\alpha_{s}{ }^{-1}\left(W_{s}+s\right) \times \prod_{i>1} B_{i}\right) \times \prod_{i \in N} B_{i}\right)\right] \times\{0\}$. Furthermore, on this set, $\left(p_{2}, p_{3}\right) \circ p_{1} \circ \theta_{s, \zeta} \circ \xi_{s, \zeta}((x, y, z), t)=\left(p_{2}, p_{3}\right) \circ p_{1} \circ \theta_{s, \zeta} \circ \xi_{s, \zeta}\left(\left(x^{\prime}, y, z\right), t\right)$ for each $y, z \in \alpha_{s}{ }^{-1}\left(W_{s}+s\right) \times \prod_{i>1} B_{i}, t \in[0,2]$, and $x$ and $x^{\prime} \in \Delta^{n}{ }_{s} \cap$ st $\left(v_{0}, M^{n-1}\right)$. 
Thus, the function $\theta_{\zeta} \circ \xi_{\zeta}$, defined only on $\left(p_{1} \circ p_{1}\right)^{-1}\left(\right.$ st $\left.\left(v_{0}, M^{n-1}\right)\right) \cap \gamma_{\zeta} \circ \beta \circ p$ $\circ \bar{g}_{\zeta}\left(Z_{2}\right)$, may be extended to a function $\sigma_{\zeta}$ on $\gamma_{\zeta} \circ \beta \circ p \circ \bar{g}_{\zeta}\left(Z_{2}\right)$ by setting

$$
\begin{array}{r}
\sigma_{\zeta}(x)=\theta_{\zeta} \circ \xi_{\zeta}(x) \text { if } p_{1} \circ p_{1}(x) \in \operatorname{st}\left(v_{0}, M^{n-1}\right), \\
=\left(\left(p_{1} \circ p_{1}(x), p_{2} \circ p_{1} \circ \theta_{\zeta} \circ \xi_{\zeta}\left(\left(v_{0}, p_{2} \circ p_{1}(x), p_{3} \circ p_{1}(x)\right), p_{2}(x)\right),\right.\right. \\
\left.\left.p_{3} \circ p_{1} \circ \theta_{\zeta} \circ \xi_{\zeta}\left(\left(v_{0}, p_{2} \circ p_{1}(x), p_{3} \circ p_{1}(x)\right), p_{2}(x)\right)\right), 0\right) \\
\text { if } p_{1} \circ p_{1}(x) \in \bigcup_{s \in A} \Delta^{n} \mid s t\left(v_{0}, M^{n-1}\right) .
\end{array}
$$

The desired uniform isomorphism $h_{\zeta}$ is then $p_{1} \circ \sigma_{\zeta} \circ \gamma_{\zeta} \circ \beta \circ p$. This establishes that $Z$ is a uniform $\prod_{i \in N} B_{i}$-approximation to $M$, so, by Theorem $2, Z \times \prod_{i \in N} B_{i}$-which is uniformly isomorphic to $Z$-is uniformly isomorphic to $M \times \prod_{i \in N} B_{i}$.

To complete the proof, all that is necessary is to observe that $Z$ is uniformly isomorphic to $\prod_{i \in N} B_{i}$. This is easy, for there is a family of uniform isomorphisms $\bar{\nu}_{s}: \Delta^{n}{ }_{s} \rightarrow\left[\Delta^{n}{ }_{s} \cap \operatorname{st}\left(v_{0}, M^{n-1}\right)\right] \times[0,1]$ such that $\bar{\nu}_{s}(x)=(x, 0)$ for each $s$ in $\Delta^{n}{ }_{s} \cap$ st $\left(v_{0}, M^{n-1}\right)$ and such that $\left\{\bar{\nu}_{s}\right\}_{s \in A} \cup\left\{\bar{\nu}_{s}^{-1}\right\}_{s \in A}$ is uniformly equicontinuous. If $\lambda$ is a homeomorphism of $[-1,1] \times\{0\} \cup\{1\} \times[0,1]$ onto $[-1,1]$ such that $\lambda\left|[-1,1-\delta]=p_{1}\right|[-1,1-\delta]$, then the function $\rho$ described by

$$
\begin{aligned}
& \rho(x)=x \quad \text { if } f_{s} \circ \alpha_{s} \circ p_{1} \circ p_{2}(x) \leqq 1-\delta, \\
& =\left(p_{1} \circ \bar{\nu}_{s} \circ p_{1}(x),\left(\alpha _ { s } { } ^ { - 1 } \left(\alpha_{s} \circ p_{1} \circ p_{2}(x)-f_{s} \circ \alpha_{s} \circ p_{1} \circ p_{2}(x) \cdot s\right.\right.\right. \\
& \left.+\left[\lambda \circ\left(f_{s} \circ \alpha_{s} \circ p_{1} \circ p_{2}, p_{2} \circ \bar{\nu}_{s} \circ p_{1}\right)(x)\right] \cdot s\right), \\
& \left.\left.p_{2} \circ p_{2}(x), p_{3} \circ p_{2}(x), \ldots\right)\right) \\
& \text { if } f_{s} \circ \alpha_{s} \circ p_{1} \circ p_{2}(x) \geqq 1-\delta
\end{aligned}
$$

will be a uniform isomorphism of $Z$ onto st $\left(v, M^{n-1}\right) \times \prod_{i \in N} B_{i}$. Therefore $M \times \prod_{i \in N} B_{i}$ is uniformly isomorphic to $\prod_{i \in N} B_{i}$, and the theorem follows by induction.

THEOREM 4. If $F$ is a Fréchet space homeomorphic to its countably infinite Cartesian power and $K$ is a metric, locally finite-dimensional, simplicial complex, then each of the following implies that $K \times F$ is an $F$-manifold:

(a) $F$ is homeomorphic to a Banach space and $c(K) \leqq \mathrm{wt}(F)$;

(b) wt $(F)$ is not the least upper bound of a sequence of lesser cardinals, and $c(K) \leqq \mathrm{wt}(F)$;

(c) $C\left(\right.$ st $\left.(v, K)^{\circ}\right)<\mathrm{wt}(F)$ for each $v \in K^{\circ}$.

Proof. (a) is just Theorem 3.

(b) If $F$ is not a Banach space, then by Theorem 5.4 of [17] there is a sequence $\left\{E_{i}\right\}_{i \in N}$ of Banach spaces and a linear embedding $T$ of $F$ in $\prod_{i \in N} E_{i}$ as a closed subspace such that $p_{i} \circ T(F)=E_{\imath}$ for each $i$. It is easy to see that

$$
\mathrm{wt}(F)=\operatorname{lub}\left\{\mathrm{wt}\left(E_{i}\right) \mid i \in N\right\},
$$

so for some $k \in N$, wt $\left(E_{k}\right)=$ wt $(F)$. 
The Bartle-Graves Theorem [4] shows that because $p_{k} \circ T$ is a linear map of $F$ onto $E_{k}$, there is a homeomorphism from $F$ to $E_{k} \times \operatorname{ker}\left(p_{k} \circ T\right)$. (Actually, the statement and proof of this theorem in [4] have nothing to do with nonnormable Fréchet spaces, but the simplified statement and proof of that part needed here $\left[15,\left(3.2^{\prime \prime}(\mathrm{a}) \Rightarrow(\mathrm{b})\right)\right]$ is easily verified for Fréchet spaces.) As $F$ is by hypothesis homeomorphic to its countably infinite Cartesian power, it is homeomorphic to the product of some Fréchet spaces with countably infinitely many copies of $E_{k}$.

Theorem 3 shows that the product of $K$ with countably infinitely many copies of $E_{k}$ (or $F$, if $F$ is a Banach space) is a manifold modelled on the product of those copies of $E_{k}$ (or $F$ ). Thus, $K \times F$ is a manifold modelled on $F$.

(c) Here, one proceeds as in case (b), but considers each vertex-star of $K$ separately. For a vertex $v$ of $K$, either $F$ is a Banach space with wt $(F)>C\left(\right.$ st $\left.(v, K)^{\circ}\right)$, or $F$ is not a Banach space but is homeomorphic to the product of some Fréchet space with a Banach space $E_{v}$ of weight greater than $C\left(\operatorname{st}(v, K)^{\circ}\right)$.

COROLlary 1. If $K$ and $L$ are two metric, locally finite-dimensional, simplicial complexes of the same homotopy type and $E$ is a Banach space homeomorphic to its countably infinite Cartesian power such that $K$ and $E$ and $L$ and $E$ each satisfy at least one of the conditions (a), (b), and (c) of Theorem 4 , then $K \times E$ is homeomorphic to $L \times E$.

Proof. By Theorem 4, $K \times E$ and $L \times E$ are $E$-manifolds of the same homotopy type. A theorem of David Henderson [12] shows them to be homeomorphic.

The next lemma was suggested to the author by Israel Berstein and David Henderson.

LEMMA 6 (BERSTEIN AND HENDERSON). Every simplicial complex $K$ with the weak topology has the homotopy type of a metric, locally finite-dimensional simplicial complex $L$ such that $c(L)=c(K)$.

Proof. If $K$ is a simplicial complex with the weak topology which is not locally finite-dimensional, then consider the space $M=\bigcup_{n \in N}\left(K^{n} \times[n, \infty)\right)$. When triangulated and given the weak topology, $M$ is a locally finite-dimensional complex. Furthermore, the projection $p: M \rightarrow K$ is easily seen to be a weak homotopy equivalence and, hence, by a theorem of J. H. C. Whitehead [21], a homotopy equivalence. Also, it is immediate that $c(M) \leqq c(K)$. The proof is completed by a theorem of Dowker [9] which says that under the metric topology, this set, called $L$, has the same homotopy type as $M$. Of course, if $K$ is locally finite-dimensional, then $L$ may be taken to be the set $K$ given the metric topology.

THEOREM 5. If $E$ is a Banach space homeomorphic to its countably infinite Cartesian power and $M$ is a paracompact manifold modelled on $E$, then $M$ is homeomorphic to the product of $E$ with a metric, locally finite-dimensional simplicial complex. 
Proof. It is sufficient to examine the case that $M$ is connected. Each paracompact Banach manifold is dominated by a simplicial complex (weak topology) [16]. Also, for any arcwise connected space, there is a simplicial complex (weak topology) weakly homotopy-equivalent to it (namely, a triangulation of the realization of its singular complex) [19]. Let $N$ be a simplicial complex dominating $M$. It may be assumed that $C\left(N^{\circ}\right) \leqq$ wt $(M)$, because all that is required is the existence of two maps $f: M \rightarrow N$ and $g: N \rightarrow M$ such that $g \circ f$ is homotopic to the identity, and the smallest subcomplex of $N$ containing $f(M)$ will do. (There can then be no more simplices of $N$ than wt $(M)$, and hence no more vertices than wt $(M)$.) Also let $K_{1}$ be a simplicial complex (weak topology) which has the same weak homotopy type as $M$. A theorem of J. H. C. Whitehead [21] shows that $K_{1}$ must be homotopyequivalent to $M$. Now, the method used by Whitehead in the proof of Theorem 24 of [22] to establish that an arcwise connected space dominated by a countable $\mathrm{CW}$-complex has the homotopy type of a countable $\mathrm{CW}$-complex immediately shows that there is a subcomplex $K_{2}$ of $K_{1}$ of the homotopy type of $M$ which has no more than wt $(M)$ simplices. The application of Lemma 6 gives a metric, locally finite-dimensional, simplicial complex $L$ of the homotopy type of $M$ with the property that $c(L) \leqq$ wt $(M)$. Since $M$ may be embedded in $E$ as an open subset by [12], wt $(M)=$ wt $(E)$. The proof is finished by applying Theorem 3 .

COROLlaRY 2. If $E$ is a Banach space homeomorphic to its countably infinite Cartesian power and $M$ is a paracompact manifold modelled on $E$ which has the homotopy type of a simplicial complex $K$ such that $c(K)<\mathrm{wt}(E)$, then for any infinite cardinal $\aleph$ with $c(K) \leqq \aleph \leqq w t(E)$, there is a pair, $F_{1}$ and $F_{2}$, of Fréchet spaces and $a$ metric, locally finite-dimensional, simplicial complex $L$ such that $c(L) \leqq c(K), L \times F_{1}$ is an $F_{1}$-manifold, wt $\left(F_{1}\right)=\aleph$, and $M$ is homeomorphic to $\left(L \times F_{1}\right) \times F_{2}$.

Proof. If $\aleph=$ wt $(E)$, then $F_{2}$ may be $E$, for $i=1,2$, and the proof reduces to that of Theorem 5 .

If $\aleph<$ wt $(E)$, let $E^{\prime}$ be a closed, linear subspace of $E$ with weight equal to $\aleph$. Now, consider a countably infinite set $\left\{E_{i}\right\}_{i \in N}$ of copies of $E$ and in each let $E_{i}^{\prime}$ be a copy of $E^{\prime}$. Let $F_{1}=\prod_{i \in N} E_{i}^{\prime}$ and $F_{2}=\left(\prod_{i \in N} E_{i}\right) / F_{1}$. Then $E$ is homeomorphic to $F_{1} \times F_{2}$.

If $\boldsymbol{N}$ is not the least upper bound of a countable set of lesser cardinals, then $L \times F_{1}$ is, by Theorem 4 , an $F_{1}$-manifold, where $L$ is a metric, locally finite-dimensional, simplicial complex of the same homotopy type as $K$ with $c(L) \leqq c(K)$. On the other hand, if $\boldsymbol{N}$ is the least upper bound of a countable set of cardinals less than it, then the method used in the proof of Theorem 5 shows that $L$ may be chosen to satisfy (c) of Theorem 4 with respect to $F_{1}$.

As $L \times F_{1}$ is an $F_{1}$-manifold, $\left(L \times F_{1}\right) \times F_{2}$ is an $E$-manifold and homeomorphic to $M$ by [12].

A restatement of the above is 
Corollary 2'. Let $E$ be a Banach space, and denote by $F$ the countably infinite product of $E$ with itself. If $M$ is a paracompact manifold modelled on $F$ with the homotopy type of a simplicial complex $K$ with $c(K) \leqq \mathrm{wt}(F)$, then there is a closed submanifold $N$ of $M$ modelled on a closed subspace $F_{1}$ of $F$ such that $M$ is homeomorphic to $N \times\left(F / F_{1}\right)$ and $\mathrm{wt}\left(F_{1}\right)=c(K)$.

COROllaRY 3. If $E$ is a Banach space homeomorphic to its countably infinite Cartesian power, then each paracompact E-manifold $M$ with the homotopy type of a countable simplicial complex is homeomorphic to the product of $E$ with a closed, separable, Hilbert submanifold of $M$.

Proof. By Lemma 6, there is a metric, locally finite-dimensional, countable, simplicial complex $L$ such that $L$ has the homotopy type of $M$, and by Theorem 4 , $L \times E$ is a manifold. Henderson's theorem [12] yields that it is homeomorphic to $M$.

Let $F$ be a separable, infinite-dimensional, closed linear subspace of $E$. The Bartle-Graves Theorem [4] shows that $E$ is homeomorphic to $F \times(E / F)$. Now, all separable, infinite-dimensional, Fréchet spaces are homeomorphic to a Hilbert space $H$ [2], so $E$ is homeomorphic to $H \times(E / F)$. By Theorem $4, L \times H$ is an $H$ manifold, so $M$ is homeomorphic to $(L \times H) \times(E / F)$, and this homeomorphism identifies $L \times H$ with the inverse image of $(L \times H) \times\{0\}-$ a closed submanifold of $M$.

\section{REFERENCES}

1. R. D. Anderson, Topological properties of the Hilbert cube and the infinite product of open intervals, Trans. Amer. Math. Soc. 126 (1967), 200-216. MR 34 \#5045.

2. R. D. Anderson and R. H. Bing, A complete elementary proof that Hilbert space is homeomorphic to the countable infinite product of lines, Bull. Amer. Math. Soc. 74 (1968), 771-792. MR 37 \#5847.

3. W. Barit, Small extensions of small homeomorphisms, Notices Amer. Math. Soc. 16 (1969), 295. Abstract \#663-715.

4. R. G. Bartle and L. M. Graves, Mappings between function spaces, Trans. Amer. Math. Soc. 72 (1952), 400-413. MR 13, 951.

5. C. Bessaga, Topological equivalence of unseparable reflexive Banach spaces. Ordinal resolutions of identity and monotone bases, Bull. Acad. Polon. Sci. Sér. Sci. Math. Astronom. Phys. 15 (1967), 397-399. MR 36 \#4321.

6. C. Bessaga and V. L. Klee, Every non-normable Fréchet space is homeomorphic with all of its closed convex bodies, Math. Ann. 163 (1966), 161-166. MR 34 \#1826.

7. C. Bessaga and A. Pełczyński, Some remarks on homeomorphisms of F-spaces, Bull. Acad. Polon. Sci. Sér. Sci. Math. Astronom. Phys. 10 (1962), 265-270. MR 25 \#3344.

8. - Estimated extension theorem, Fund. Math. 69 (1970), 153-190.

9. C. H. Dowker, Topology of metric complexes, Amer. J. Math. 74 (1952), 555-577. MR 13, 965.

10. D. W. Henderson, Infinite-dimensional manifolds are open subsets of Hilbert space, Topology 9 (1969), 25-33. MR 40 \#3581.

11. - Open subsets of Hilbert space, Compositio Math. 21 (1969), 312-318. MR 40 \#4975.

12. - Stable classification of infinite-dimensional manifolds by homotopy type, Invent. Math. 12 (1971), 48-56. 
13. V. L. Klee, Some topological properties of convex sets, Trans. Amer. Math. Soc. 78 (1955), 30-45. MR 16, 1030.

14. A. H. Kruse, Badly incomplete normed linear spaces, Math. Z. 83 (1964), 314-320. MR 29 \#3859.

15. E. Michael, Continuous selections. I, Ann. of Math. (2) 63 (1956), 361-382. MR 17, 990.

16. R. S. Palais, Homotopy theory of infinite dimensional manifolds, Topology 5 (1966), 1-16. MR 32 \#6455.

17. H. H. Schaefer, Topological vector spaces, Macmillan, New York and London, 1966. MR 33 \#1689.

18. H. Toruńczyk, Skeletonized sets in complete metric spaces and homeomorphisms of the Hilbert cube, Bull. Acad. Polon. Sci. Sér. Sci. Math. Astronom. Phys. 68 (1970), 119-126.

19. S. Weingram, On the triangulation of the realization of a semisimplicial complex, Illinois J. Math. 12 (1968), 403-413. MR 38 \#6579.

20. J. E. West, Infinite products which are Hilbert cubes, Trans. Amer. Math. Soc. 150 (1970), $1-25$.

21. J. H. C. Whitehead, Combinatorial homotopy. I, Bull. Amer. Math. Soc. 55 (1949), 213-245. MR 11, 48.

22. - A certain exact sequence, Ann. of Math. (2) 52 (1950), 51-110. MR 12, 43.

Department of Mathematics, Cornell University, Ithaca, New York 14850 